\title{
New epidemiological data of multiple sclerosis, the impact of psychopathological symptoms on the health-related quality of life of multiple sclerosis patients
}

\author{
Ph.D. thesis \\ Tamás István Biernacki, M.D.
}

Clinical and Experimental Neurosciences Program Doctoral School of Clinical Medicine, Faculty of Medicine, University of Szeged

Supervisor: Krisztina Bencsik, M.D., Ph.D, med. habil.

Department of Neurology, Albert Szent-Györgyi Clinical Centre, University of

Szeged

2020. 10. 13. 


\section{Table of Contents}

Original publications directly related to the $\mathrm{Ph} . \mathrm{D}$. thesis...................................................... 3

Publications (original and review) not directly related to the Ph.D. thesis .............................. 3

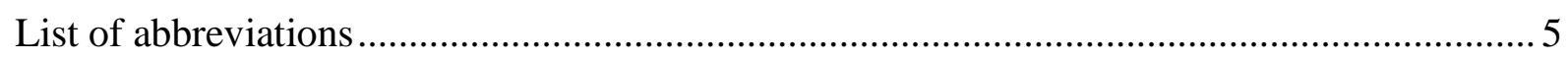

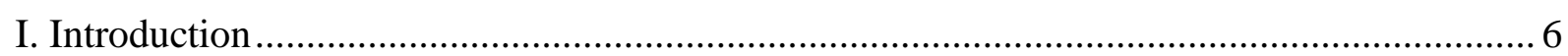

I. 1. Disease course classifications and disease activity in MS .......................................... 6

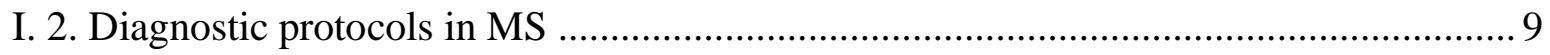

I. 3. Introduction of a novel, phenotypic classification system ....................................... 10

I. 4. Introduction of the first international guidelines on the treatment of MS .................. 12

I. 5. Health-related quality of life of MS patients ...................................................... 13

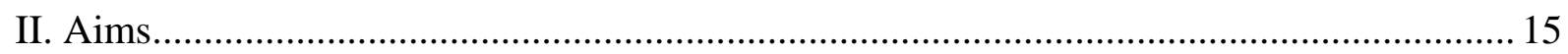

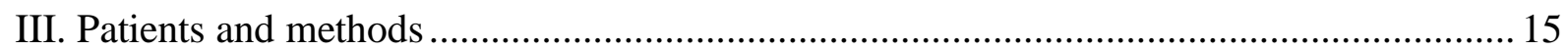

III.1. Fresh epidemiological data based on the novel phenotypic classification of MS and

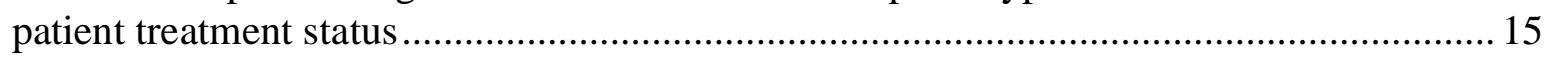

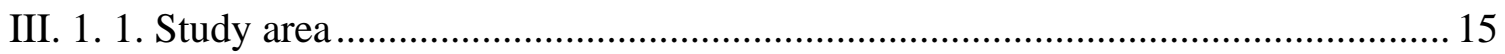

III. 1. 2. Methods, the establishment of diagnosis, follow-up examinations ................... 16

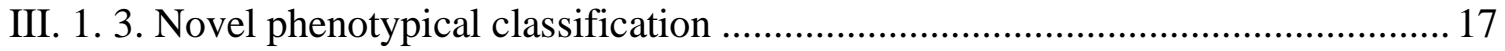

III. 1. 4. Definition of secondary progressive MS ...................................................... 18

III.2. Factors predicting and influencing the health-related quality of life of MS patients.. 19

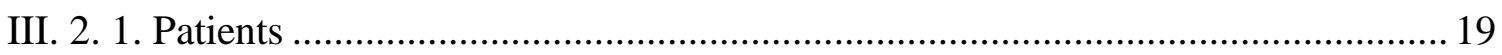

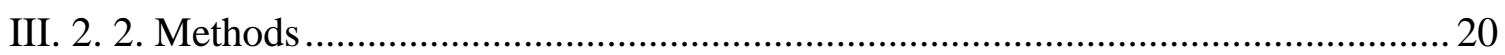

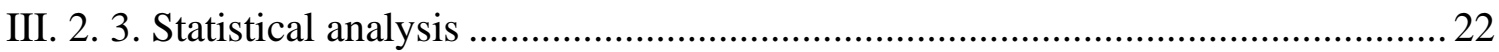

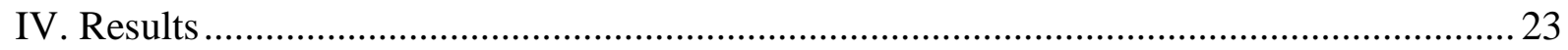

IV. 1. Fresh epidemiological data based on the novel phenotypic classification of MS and

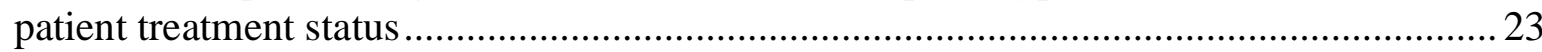

IV. 2. Factors predicting and influencing the health-related quality of life of MS patients 25

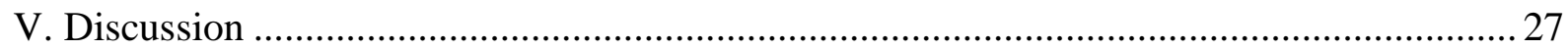

V. 1. Fresh epidemiological data based on the novel phenotypic classification and treatment status

V. 2. Factors predicting and influencing the health-related quality of life of MS patients.. 30

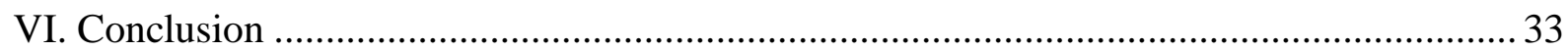

VI. 1. Fresh epidemiological data based on the novel phenotypic classification and

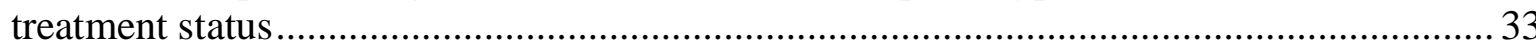

VI. 2. Factors predicting and influencing the health-related quality of life of MS patients 34 Tables .36

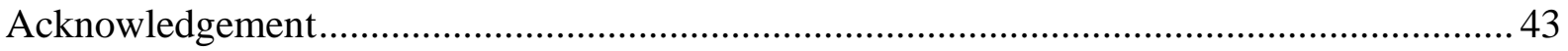

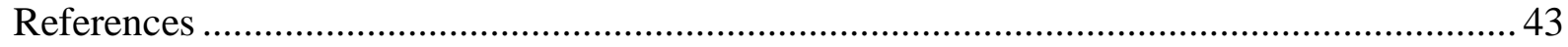

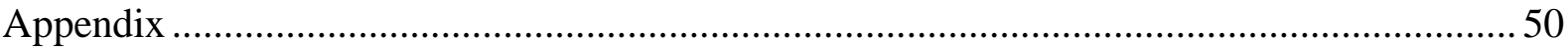


Original publications directly related to the $\mathrm{Ph} . \mathrm{D}$. thesis

Biernacki T, Sandi D, Fricska-Nagy Z, Kincses ZT, Füvesi J, Laczkó R, Kokas Z, Klivényi P, Vécsei L, Bencsik K. Epidemiology of multiple sclerosis in Central Europe, update from Hungary. Brain Behav. 2020 May;10(5)

IF: 2.091

Biernacki T, Sandi D, Kincses ZT, Füvesi J, Rózsa C, Mátyás K, Vécsei L, Bencsik K. Contributing factors to health-related quality of life in multiple sclerosis. Brain Behav. 2019 Dec;9(12)

IF: 2.091

\section{Publications (original and review) not directly related to the Ph.D. thesis}

Sandi D, Rudisch T, Füvesi J, Fricska-Nagy Z, Huszka H, Biernacki T, Langdon DW, Langane É, Vécsei L, Bencsik K. The Hungarian validation of the Brief International Cognitive Assessment for Multiple Sclerosis (BICAMS) battery and the correlation of cognitive impairment with fatigue and quality of life. Mult Scler Relat Disord. 2015 Nov;4(6):499-504. IF: 1.150

Sandi D, Biernacki T, Szekeres D, Füvesi J, Kincses ZT, Rózsa C, Mátyás K, Kása K, Matolcsi J, Zboznovits D, Burány Z, Langane É, Vécsei L, Bencsik K. Prevalence of cognitive impairment among Hungarian patients with relapsing-remitting multiple sclerosis and clinically isolated syndrome. Mult Scler Relat Disord. 2017 Oct;17:57- 62. IF: 3.199

Sandi D, Bereg E, Biernacki T, Vörös E, Klivényi P, Bereczki C, Vécsei L, Bencsik K. Pediatric multiple sclerosis and fulminant disease course: Features and approaches to treatment - A case report and review of the literature. J Clin Neurosci. 2018 Jul;53:13- 19. IF: 1.640

Bencsik K, Sandi D, Biernacki T, Kincses Z, Füvesi J, Fricska-Nagy Z, Vécsei L. The Multiple Sclerosis Registry of Szeged. Ideggyogy Sz. 2017 Sep 30;70(9-10):301-306. IF: 0.322

Biernacki T, Bencsik K, Sandi D, Vécsei L. Alemtuzumab Therapy 2017. Ideggyogy Sz. 2017 Nov;70(11-12):371-380.

IF: 0.322

Biernacki T, Bencsik K, Kincses ZT, Sandi D, Fricska-Nagy Z, Faragó P, Vécsei L. Change of therapeutic algorithm in sclerosis multiplex based on two case reports. Ideggyogy Sz. 2017 Nov;70(11-12):381-387.

IF: 0.322

Biernacki T, Sandi D, Bencsik K, Vécsei L. Kynurenines in the Pathogenesis of Multiple Sclerosis: Therapeutic Perspectives. Cells. 2020;9(6):1564.

IF: 4.366 
Biernacki T, Sandi D, Bencsik K, Vécsei L. Medicinal Chemistry of Multiple Sclerosis: Focus on Cladribine. Mini Rev Med Chem. 2020;20(4):269-285.

IF: 2.733

Cumulative impact factor: 18.236 


\section{List of abbreviations}

A-NP - active-not progressive (patient showing disease without progression)

A-NT - active-not treated (patients showing disease activity without receiving treatment)

A-P - active-progressive (patient showing disease and progression at the same time)

A-T - active-treated (patients showing disease activity despite receiving treatment)

BDI II - Beck Depression Inventory II

BICAMS - Brief International Cognitive Assessment for MS

BVMT-R - Brief Visuospatial Memory Test-Revised

$\mathrm{CI}$ - cognitive impairment

CIS - clinically isolated syndrome

CVLT-II - California Verbal Learning Test-II

$\mathrm{CSF}$ - cerebrospinal fluid

DMT - disease-modifying treatment

EDSS - expanded disability status scale

FIS - Fatigie Impact Scale

HADMT - highly active disease-modifying treatment

HRQoL - health-related quality of life

MS - Multiple sclerosis

MSQoL-54 - Multiple Sclerosis Quality of Life-54

NA-NP - not active-not progressive (patient not showing disease activity nor progression)

NA-NT - not active-not treated (patient not showing disease activity without receiving treatment)

NA-P - not active- progressive (patient not showing disease activity while showing progression)

NA-T - not active-treated (patient not showing disease activity while receiving treatment)

PLS - partial least squares regression

PPMS - primary progressive MS

PRMS - progressive-relapsing MS

PwMS - patients with MS

RIS - radiologically isolated syndrome

$\mathrm{RR}$ - relapsing-remitting

RRMS - relapsing-remitting MS

SDMT - Symbol Digit Modalities Test 
SMPC - summary of product characteristics

SMR - standardized mortality ratio

SPMS - secondary progressive MS

VIP score - variable importance of projection score

\section{Introduction}

Multiple sclerosis (MS), although considered as a rare disease, is the most common chronic, autoimmune, demyelinating, and neurodegenerative disease of the central nervous system. MS is characterized by continuous inflammation, demyelination, remyelination, axonal and bloodbrain-barrier damage throughout its natural history, though the intensity and presence of these processes change through time, as the disease progresses [1-3]. The name of the disease originates from the very first pathological findings [4] of Charcot, who summarized previous reports and added his own clinical and pathological observations. Charcot named the disease „sclerose en plaques”, he was the first one to recognize and classify multiple sclerosis as a distinct disease in 1868. MS proved to be the second most common cause (after traumatic injury) of permanent disability among young adults [5], which gravely impacts and worsens the health-related quality of life of patients; compared to other autoimmune diseases, MS patients perceived their health-related quality of life to be the worst [6]. Furthermore, in the past few years, several psychopathological symptoms of MS have been shown to develop insidiously and progress gradually even from the beginning of the disease, many of which have been documented to alter the patients' health-related quality of life. Additionally, in recent years new diagnostic criteria, phenotypic disease course classification, and treatment protocol have been published, the therapeutic palette has expanded considerably, nowadays there is at least one drug available for every disease course. Adjacent to the introduction of new disease-modifying therapies, a novel standpoint is gaining ground regarding the therapeutic strategy of MS.

\section{1. Disease course classifications and disease activity in MS}

The most distinctive clinical attributes of MS (other than the plethora of diverse neurological symptoms it can cause, it is called the "lupus of neurology" for a reason) are the progression and the activity of the disease. Disease progression just as activity can be defined over an ' $\mathrm{x}$ ' amount of time (usually 6 months or 1 year), both can be either radiological or clinical. Clinical progression is defined as the objectively documented, steadily increasing accumulation of neurological symptoms/disability without unequivocal recovery, however, transient fluctuations and stability in a patient's state can occur [7]. As for radiological progression, no standardized imaging markers are established at the time this thesis is being written; however, 
brain volume loss (ie. atrophy), the increasing number and volume of $\mathrm{T} 1$ hypointense lesions, and other MRI sequences are being studied which currently are not part of the everyday routine practice [7].

Figure 1.: brain MRI scans of a patient with secondary progresive MS.

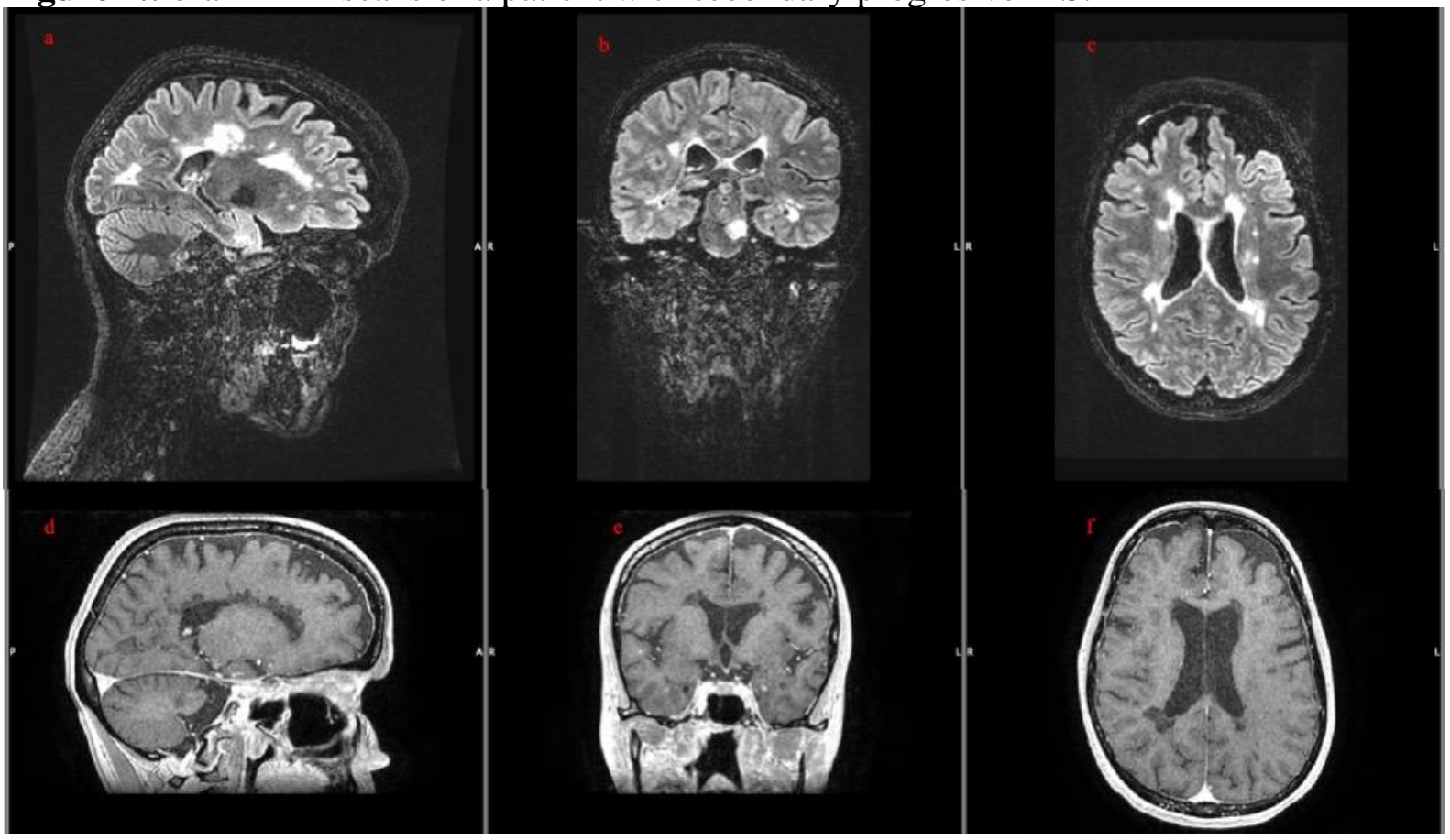

Note: a-b-c, saggital, coronal and axial T2 FLAIR images show a high T2 hyperintense lesion load; d-e-f, saggital, coronal and axial contrast-enhanced images show several black holes, but no contrast-enhancing, fresh lesions. At the time of the scans the patient showed no disease activity.

Figure 2.: The brain MRI scans of the same patient during a relapse.

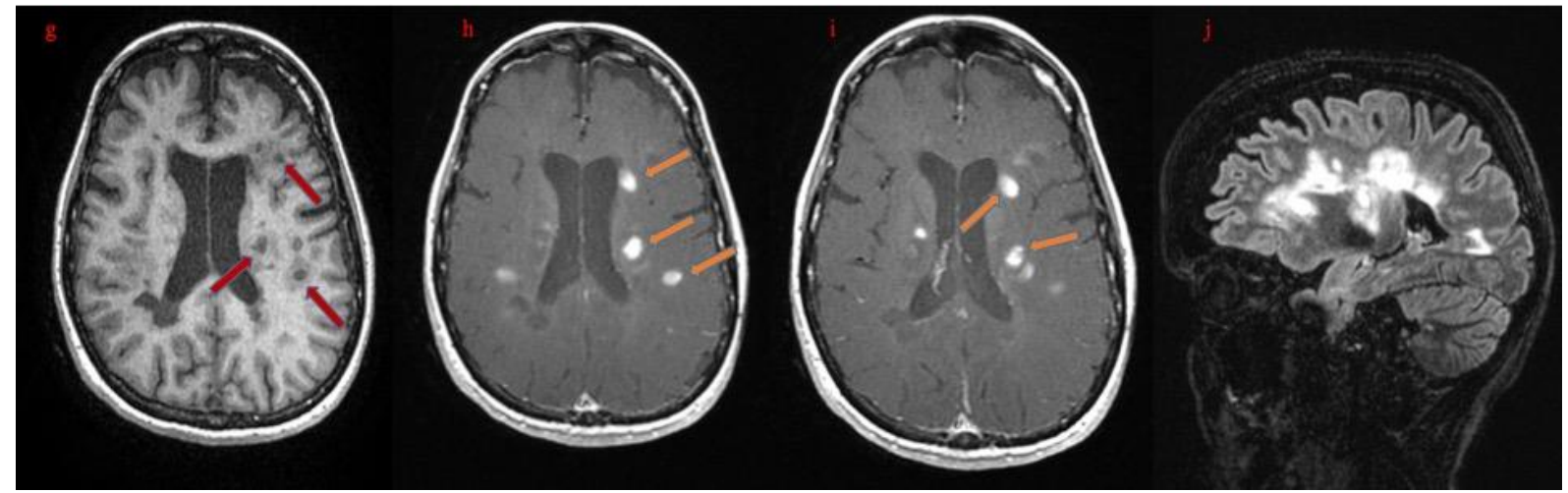

Note: g, native axial T1 image, the red arrows indicate black holes; h,i, axial contrast-enhanced $\mathrm{T} 1$ images, the orange arrows indicate fresh, contrast-enhancing $\mathrm{T} 1$ hyperintense lesions; $\mathrm{j}$, saggital T2 FLAIR image showing unequivocally enlarging T2 hyperintense lesions in comparison with image ,a".

Clinical disease activity can be described as the acute or sub-acute onset of new or the worsening of an already sustained neurological dysfunction which is followed by a complete or partial recovery, in the absence of fever or infection [7]. Radiological disease activity is 
defined as the emergence of new/unequivocally enlarging T2 hyperintense, and/or contrastenhancing T1 hyperintense lesions on MRI images [7]. Based on these two attributes in order to standardize terminology, provide clarity and consistency in defining patient groups for studies and clinical trials, furthermore to unify, and to make communications between clinicians comprehensible, concise and accurate Lublin and his colleagues defined the „classical” disease courses of MS in 1996 [8]. In total 4, very distinctive clinical courses have been described. The most frequently observed form of MS is characterized by the presence of clearly defined episodes of acute worsening of neurologic function (relapses) followed by full or partial recovery, with no detectable disease progression between relapses. This form was termed relapsing-remitting MS (RRMS). Secondary progressive form of the disease (SPMS) could be viewed as an epilogue to RRMS, as the consensus definition defined this disease course as ,an initial RR disease course followed by progression with or without occasional relapses, minor remissions, and plateaus". In 1996 no clear-cut margins were defined where the relapsingremitting phase ended and the secondary progressive phase began. This resulted in many controversies among clinicians and in patient recruitment into studies, a problem, which was amended 20 years later by Lorscheider et al (see later). Primary progressive MS (PPMS, which can be seen in 10-15\% of the patients) in contrast to RRMS is described by a constant, gradual worsening of symptoms already from disease onset and the complete absence of relapses (although minor fluctuations in the patients' state were allowed by the consensus definition). The $4^{\text {th }}$ and rarest of the clinical forms was coined progressive-relapsing MS (PRMS). It was defined as a progressive disease from onset (much similar to PPMS), but with the unequivocal presence of acute relapses, with full or partial recovery, furthermore periods between the relapses are characterized by a continuous progression of symptoms [8]. Along with the clinical courses discerned by disease activity two other „entities” were defined based on the severity of the symptoms. Benign MS was described as a „disease in which the patient remained fully functional in all neurologic systems 15 years after disease onset". In routine clinical practice this meant that the patients did not show any clinically meaningful disease activity and/or progression, their expanded disability status scale (EDSS) score did not surpass 3 points after 15 years. A course termed malignant MS was also defined, which is the exact opposite of benign MS. The consensus definition for malignant MS was ,a disease with a rapid progressive course, leading to significant disability in multiple neurologic systems or death in a relatively short time after disease onset", the exact time frame to which relatively short referred to was left undefined, however. These clinical course descriptions had become the gold standard of everyday MS practice and clinical research until they got superseded by the first revision in 
2013 [9], when sufficient progress and new insights became available to recommend changes to the old descriptions. Even though the diagnosis of MS can and should be made on clinical grounds even nowadays, the advances made in imaging and other paraclinical studies and the 2010 revisions to the diagnostic criteria of MS (see later) helped clarify some unclear cases, meanwhile raised doubts about the diagnosis of other patients presenting with MS-like symptoms. This invoked the need to expand the original disease courses with two new candidates; the 2013 revisions introduced „clinically isolated syndrome” (CIS) and „radiologically isolated syndrome” (RIS) as potential new phenotypes. CIS got classified as a new, distinct MS phenotype, it was defined as the first clinical presentation of a disease that shows characteristics of inflammatory demyelination, that could be MS, but has yet to fulfill the criteria of dissemination in time. RIS, on the other hand, was described as a state in which incidental imaging findings are suggestive of inflammatory demyelination, but the patient lacks any clinical signs or symptoms, and as such only imaging findings raise the suspicion of MS, greatly depending on the morphology and location of the detected MRI lesions. Therefore patients who would be classified as RIS with no obvious clinical signs or symptoms suggestive of MS were advised to be followed prospectively, consensus expert opinion at the time concluded that RIS should not be considered a distinct MS phenotype. This decision was reinforced later, as data in support of RIS being a distinct phenotype was lacking and the concept of RIS was completely omitted from the disease courses by future phenotypic classifications.

\section{2. Diagnostic protocols in MS}

Contrary to the future-proof clinical course definitions the diagnostic protocols evolved greatly along the years and have been revamped quite often as increasingly more technological advancements became widely available in the everyday clinical practice. In the past decades, several protocols and criteria were published and had been implemented into routine clinical and scientific practice to diagnose MS. After careful review of the historical and clinical symptomatology of MS in 1983 Poser et al. [10] made comprehensive, objective, exact, and up-to-date diagnostic criteria that integrated clinical, laboratory, and imaging findings. It introduced several novel concepts such as attacks, clinical and paraclinical evidence, also omitted the age restriction from the previously ubiquitously used Schumacher requirements [11]. It retained the foundation of the previous protocol, namely the need to demonstrate the disease's dissemination in time and space in order to verify the diagnosis of MS. Based on clinical and paraclinical evidence it distinguished 5 diagnostic categories; clinically definite, 
laboratory-supported definite, clinically probable, and laboratory-supported probable diagnosis of MS, and if no evidence was in support of MS, no MS. The great significance of the protocol was that it broke with the various vague and obscurely phrased diagnostic protocols published in prior decades, many of which relied on discrepant terminology and laid too much emphasis on the examiner's subjective judgment. Thus the criteria of Poser replaced the most commonly used Schumacher criteria [11], rapidly became and remained the gold standard in the diagnosis of MS for almost two decades. They eventually became obsolete in 2001, when it was superseded by the first diagnostic protocol of McDonald [12]. Similarly to the Poser criteria, they maintained the requirement to demonstrate the dissemination of lesions attributed to MS in time and space, however, the number of diagnostic categories based on clinical and paraclinical (MRI and cerebrospinal fluid [CSF] examination) findings got reduced to 3 possibilities; „MS”, „possible MS” and „not MS”. The first McDonald criteria kept the possibility to diagnose MS based solely on clinical grounds, however, for the first time, it proposed that in the absence of definite clinical evidence (ie. a patient had less than two verified attacks and less than two objective lesions in separate anatomical regions) MRI and/or CSF findings can serve as surrogates for the confirmation of dissemination in space and/or time to diagnose MS. These advancements facilitated the diagnosis of MS in patients who presented with solely one attack meanwhile increased the sensitivity of the criteria compared to the Poser criteria without compromising specificity. Since 2001 three updates of the McDonald diagnostic protocol have been published [13-15], each of which further boosted the sensitivity, and allowed for a more rapid diagnosis while maintaining specificity. The most recent revisions were published in 2017 [15], according to these, the diagnosis of clinically definitive multiple sclerosis can now be made even in patients whose disease previously was classified as CIS, based on solely one clinical event supported by an MRI and/or CSF examination. Furthermore, new evidence suggests that the baseline MRI scan's purpose points far beyond the sole establishment of the diagnosis, as it was shown that future disease activity could be predicted from the baseline MRI parameters [16].

\section{3. Introduction of a novel, phenotypic classification system}

A new, phenotype-based classification has also been introduced recently [7], in contrast to the older classification system it employs a markedly different approach to the disease courses. It maintained much of the basic attributes of the originally defined disease courses, however, it lays a much greater emphasis on the activity of the disease and the gradual worsening of symptoms in order to categorize the disease into different phenotypes. It recognizes two 
distinctive, major courses of MS, the relapsing-remitting (previous RR and CIS disease courses) type, and the progressive type [7]. Depending on the clinical and imaging presentation, a patient's disease classified as CIS can be stable and show no activity or can show clinical and/or radiological activity, in which case the disease is considered relapsing-remitting. Similarly, patients with a RR disease can be stable or can show clinical and/or radiological activity. Likewise, patients with progressive diseases can be separated into two groups whether radiological and/or clinical activity is present (ie. progressive disease with, or without activity). These two categories can further be split based on the presence or the lack of continuous worsening of symptoms (ie. progressive disease type with, or without progression) [7]. As mentioned previously, when disease courses were categorized based solely on the presence/lack of relapses, the presence/lack of continuous worsening between relapses and on the EDSS scores [17] no clear cut margin was defined where the relapsing-remitting phase ended and secondary progressive phase (most commonly still with relapses) began [10], there was an overlap between the EDSS scores of the two disease types. The EDSS score of RR patients traditionally ranged from 0 to 5.5, whereas it laid between 3.5 and 10.0 for SP patients. Large scale studies exploring the natural history of the disease [18], and epidemiological studies employing long-term follow up have shown, that no matter how long it took a patient from disease onset to reach the turning point between the relapsing and progressive state, the disease advances roughly with the same speed in all patients thereafter. This, among other facts, was the causes that first has lead the scientific community to question the viability of the aforementioned „benign” disease course, and eventually has resulted in the complete abandonment of the concept $[18,19]$. Other reasons for the omission of „benign” MS were the results of studies, which have shown that patients with a „benign” disease course are just as burdened by fatigue, depression, anxiety, various psychiatric conditions and show signs of cognitive dysfunction as are patients who exhibit more disease activity resulting in higher EDSS scores [20-23]. One of the important features of the new phenotypic classification system, therefore, is the fact that it categorizes MS based on disease activity rather than raw EDSS score, thus it considers the psychopathological symptoms of the patients as well, which do not show up in the EDSS score, but can be signals of disease activity nonetheless.

Irreversible axonal injury and transition into the progressive phase of the disease have been shown to occur approximately at an EDSS score of 4 [24], our therapeutic window to stabilize a patient's illness and to prevent the patient from reaching the point of no return is before this [25]. It is not an impossible mission to achieve this with the disease-modifying treatments 
(DMT) currently at our disposal, as compared to the natural history of MS it is now possible to significantly flatten the disease activity curve, thus we are capable to remarkably prolong the time spent in good health from disease onset before irreversible axonal injury happens. Recent data has shed light on the fact that the socio-economical and financial burden an MS patient places on the healthcare provider and society is greatly dependent not just on the amount of the patients, but on the patient's disease type as well. Secondary progressive MS patients were shown to impose almost twice the burden on society than RRMS patients do [26]. Interestingly the bulk of the cost did not come from medical expenses but from the direct non-medical costs and productivity loss, which are the direct consequence of the early retirement of patients with SPMS [26].

\section{4. Introduction of the first international guidelines on the treatment of MS}

Not only the diagnostic criteria, disease course classifications had been revamped in recent years, but the very first, up-to-date, comprehensive, and exact guideline has been also published on the pharmacological treatment of MS patients [27]. It broke with the previous escalative approach, where the treatment of an MS patient was commenced with a less potent, so-called "platform" drug (interferons, glatiramer acetate, dimethyl fumarate, teriflunomide), which, if turned out to be inefficient in halting the disease, could be switched onto more potent a DMT. The novel therapeutic guideline advocates against this practice, it recommends the treatment of every patient as soon as possible, with disease-modifying treatments matching the patient's disease activity, advises the use of highly active DMTs (HAMDT - fingolimod, natalizumab, ocrelizumab, cladribine, alemtuzumab, and mitoxantrone) already as the first choice of treatment for patients with highly active disease. In order to achieve this, to be able to provide a personalized treatment tailored to the patient's disease activity it is imperative to constantly monitor the patient and react to the slightest signs of progression and/or disease activity. The new phenotypic classification aids the clinician in this goal by giving a pivot in assessing the disease activity of their patients and therefore helping with choosing the most suitable DMT for them, also alerting the physician when to change treatment, to recognize if a given DMT has become inefficient. 
Figure 3.: Treatment strategy of MS according to the new therapeutic guidelines.

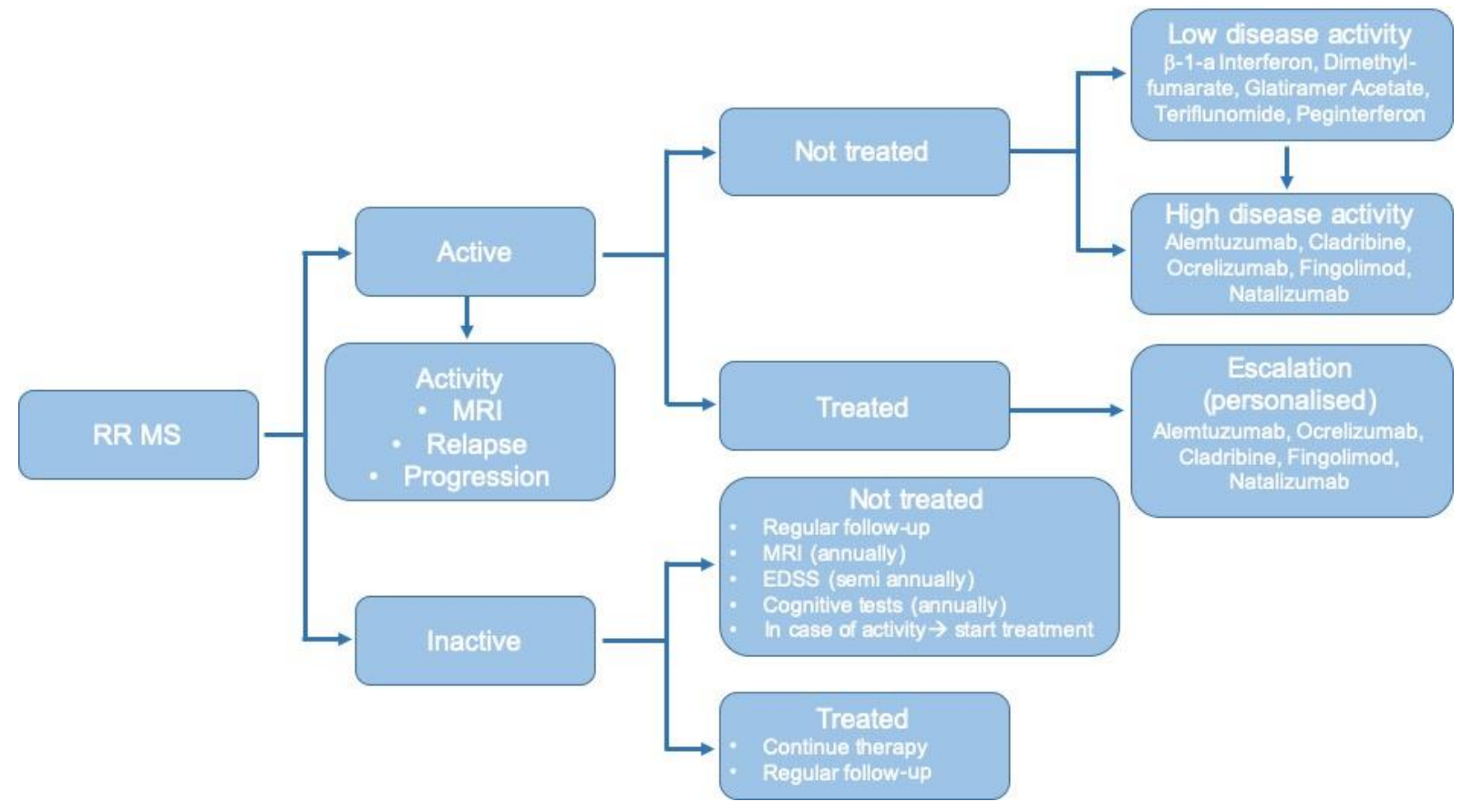

Due to the previously mentioned advances in the diagnosis and classification of MS fresh, upto-date, comparable epidemiological data using exact definitions and the aforementioned principles are needed which comply with the requirements of the modern-day. This data is essential not just to the healthcare provider in order to assess the therapeutic, medical, and financial needs of patients with MS on a population basis [28], but also crucial for clinicians to re-evaluate the medical needs of many patients who were previously diagnosed with an RR disease type, but now fall into the secondary progressive disease category according to the new classification system, therefore require a fundamentally different therapeutic approach and treatment [24]. To be able to provide a picture of disease development in a given area over time that reflects the results of the DMTs, therapeutic and diagnostic protocols used we need regularly updated, fresh, good quality - preferably using information extracted from specialized registries - epidemiological data that are based on the forever respective therapeutic and diagnostic protocols.

\section{5. Health-related quality of life of MS patients}

In the ,early days”, when the life expectancy of the general population did not exceed the life expectancy of an MS patient by much MS was believed to only influence the patients' quality of life, but do not affect the lifespan and mortality. This point of view was held for more than a century after the first detailed description of the disease was made by Charcot in 1868. This approach was disproved when the first report from Scotland [29] in the late '80s stated that 
more than half of the MS patients had died due to MS-related causes, a finding which was corroborated by many following studies [30-32]. Subsequently, MS was shown to not only alter the patients' cause of death but to drastically decrease their life expectancy as well [31, 33, 34]. MS proved to be a huge mortality risk for the patients beyond the reduced life expectancy, patients with MS (PwMS) were shown to have roughly 2.5 to 4 times greater standardized mortality ratio (SMR) compared to the general population. Primary progressive form of the disease was associated with an even greater rise in the patients' SMR than RRMS, furthermore, patients with disease onset before the age of 18 were found to have the highest SMR [30, 3335]. When the connection between MS and SMR was investigated in retrospective studies and the observation period was fragmented and shortened to encompass only decades an interesting trend was discovered, however. The highest SMR values were seen in the '50s and ' 60 s from which point it showed a constant decrease. After the introduction of disease-modifying treatments, the SMR of MS patients fell below 1, meaning that MS patients were more likely to survive longer than their life expectancy at birth [34]. As increasingly more DMTs became available and a consecutive rise in survival followed a new, unmet medical need rose among MS patients. Not only the net amount of gained life years began to matter, but the quality of those years as well. As expected, several studies have shown MS patients' health-related quality of life (HRQoL) to be worse compared to the general population, also suicide rates were shown to be higher in PwMS than in the general population, especially at the time of diagnosis, not at symptom onset [36-38]. Furthermore when compared to patients suffering from other autoimmune conditions MS patients were found to have the lowest perceived HRQoL [6]. Over the past years, several factors have been identified to influence the patients HRQoL; lower household income, higher EDSS score, a lower score on the 9-hole-peg test, weaker coping capacity, and a more debilitating, more progressive disease course, the presence of psychopathological symptoms, just to name a few, have all been linked to a lower HRQoL, which in turn has been shown to foreshadow worse survival in PwMS [17, 39-42]. As halting the physical symptoms and progression of disease became possible with DMTs an increased amount of interest turned to the research of other, previously unmeasured, and neglected symptoms of MS. In the past two decades, it became apparent, that besides physical symptoms the overwhelming majority of PwMS suffer from cognitive and psychological symptoms, which can develop at any stage of the disease $[43,44]$, the lifetime prevalence of depression, cognitive impairment (CI) and fatigue turned out to be about $50 \%, 43 \%-70 \%$, and over $90 \%$, respectively [45-47]. 
In recent years several studies have come to the same conclusion, that the psychological symptoms accompanying MS have a serious impact on the HRQoL of the patients, which effect is independent of their physical state. CI was shown to be one of the most crucial factors in patients becoming unemployed and leading to several other serious burdens [48], also male patients were found to be more prone to developing CI [47]. Furthermore, fatigue and depression were both demonstrated to be individual factors that heavily worsen the HRQoL of PwMS in almost all domains of life [42, 49-51]. Even though it was suggested a relatively long time ago [49], that the aforementioned psychological aspects may have an even greater role in determining the HRQoL of PwMS than their physical state does, the independent contribution and the actual level of impact of these known, and several other potential influencing factors on the HRQoL of PwMS is only scarcely, if ever investigated.

\section{Aims}

Our aims were:

1. To review the prevalence and incidence of MS in a previously surveyed demographic region, to provide fresh epidemiological data using the latest diagnostic criteria and the novel phenotypic classification system.

2. To present data about the disability, treatment status, and disease activity of these MS patients.

3. To investigate the independent effect and level of influence of various clinical, sociodemographic, and psychological aspects on the HRQoL of MS patients.

4. To determine which are the most important contributing factors influencing MS patients' HRQoL.

5. To explore the presence of potential differences between the sexes, considering that there are known differences between male and female patients regarding the prognosis and symptoms of MS.

\section{Patients and methods}

\section{III.1. Fresh epidemiological data based on the novel phenotypic classification of MS and patient treatment status}

\section{1. 1. Study area}

The MS outpatient clinic of the Department of Neurology of the University of Szeged is providing healthcare for MS patients from Csongrád-Csanád-, Békés- and Bács-Kiskun county, 
thus is responsible for the treatment of approximately 1,231,000 inhabitants [52]. The surveyed region was restricted to Csongrád-Csanád county from the clinic's tri-county obligation to provide in-area care. Csongrád-Csanád county is located in the south-eastern region of Hungary in the temperate zone, with an area of $4262.68 \mathrm{~km}^{2}$ and a humid continental climate. On the prevalence day of January $1^{\text {st }}, 2019,399,012$ people resided in Csongrád-Csanád county (accounting for $4.08 \%$ of the total population of Hungary), of which 189,420 were males and 209,592 females according to the latest census [53].

\section{1. 2. Methods, the establishment of diagnosis, follow-up examinations}

Every patient treated by our clinic has been included in the Multiple Sclerosis Register of the Department of Neurology of the University of Szeged since its start in 1993 [54]. All of the enrolled patients' data were extracted from the MS register. All data shown represent the state of the patients on the prevalence day, disease duration was calculated from the onset of each patient's disease. The diagnosis of MS was without exception established and re-evaluated at each visit by the given patient's respective neurologist, always in adherence to the most recent available diagnostic criteria. The criteria of Poser [10] were used until 2001, in the following years always the most up-to-date McDonald criteria were applied [12-15]. Brain and, if necessary, spinal cord MRI imaging was done on a $1.5 \mathrm{~T}$ scanner until 2017, from which point brain MRI scans were conducted on a 3T scanner. Imaging was consistently done in adherence to the latest MAGNIMS guidelines [55]. After the baseline MRI examination, control MRI scans were conducted on a regular basis, always in adherence to the SMPC of the DMT a given patient was treated with, but at least annually. As a part of the diagnostic process, every patient underwent lumbar puncture to acquire CSF to be analyzed in our clinic's accredited laboratory (according to ISO 9002 standards) by the means of laser nephelometry for the quantitative determination of proteins, isoelectric focusing, and $\mathrm{IgG}$ immunoblotting for the detection of oligoclonal bands. Additionally, if required, visual evoked potential, somatosensory evoked potential, and brainstem evoked response audiometry tests were conducted. Every patient's medical history has been reviewed by four neurology specialists independently and were excluded if at least one specialist felt uncertain about the diagnosis. After the diagnosis of MS was established every patient with an RR disease type irrespective of treatment status, and patients with a progressive disease type who received immunomodulatory therapy were routinely examined every 3 months. Every other patient underwent a routine check-up at least annually. Patients with a diagnosis of CIS were advised to annually attend a routine check-up indefinitely, unless another disease responsible for their symptoms was diagnosed in the 
meantime or disease activity presented, from which point the regular visit schedule was recommended (ie. at least every 3 months). An unscheduled, urgent appointment was always provided for the patients in the event of a relapse. If indeed a relapse was the cause of the patient's symptoms before the initiation of intravenous corticosteroids, a contrast-enhanced MRI scan was always obtained. Patients who were diagnosed with CIS underwent control brain MRI examinations 3 and 6 months after the diagnosis, and yearly thereafter if no unequivocal pathology was confirmed on the control scans. Every patient with a "classical" RR disease type underwent re-evaluation for secondary progressive disease course using the objective, 3-strata criteria proposed by Lorscheider et al. [24].

The 2013 standard European population was used in calculating the standardized prevalence. The study was approved by the Ethics Committee of the Faculty of Medicine, University of Szeged (207/2015-SZTE). All participants gave their written informed consent in accordance with the Declaration of Helsinki. For the statistical analyses, SPSS version 22 was used.

\section{1. 3. Novel phenotypical classification}

In 2014 a novel, phenotype-based classification system was proposed by Lublin et al. [7] to reclassify the disease courses of MS. It breaks with the "old" classification system's principles and, in contrast, it lays a much greater emphasis on, and uses the activity of the disease (radiological and/or clinical) and the gradual worsening of symptoms (ie. disease progression) as a basis to categorize the disease into different phenotypes. Disease activity is defined individually over ' $n$ ' time; usually 1 year, can be clinical and/or radiological. Clinical disease activity is characterized by the occurrence of a relapse followed by full or partial recovery, in the absence of fever or infection or evidence of a pseudo-relapse. Radiological activity is described as the occurrence of contrast-enhancing T1 hyperintense lesions and/or new or unequivocally enlarging T2 hyperintense lesions. Disease progression is also defined over ' $n$ ' time, usually 1 year, it can be radiological and/or clinical similarly to disease activity. Clinical progression is defined as steadily increasing objectively documented neurological dysfunction/disability without unequivocal recovery. Even though no standardized imaging measures of disease progression are currently established radiological progression is proposed to be defined as an increasing number and volume of T1 hypo-intense lesions, also brain volume loss, and changes in magnetization transfer imaging and diffusion tensor imaging are under investigation [7]. 
Based on the aforementioned definitions the new classification system separates the disease into two distinct phenotypes; the relapsing-remitting type, and the progressive phenotype, further divides the phenotypes based on disease activity and progression. The patients' disease classified as CIS can be stable and show no activity, or can be clinically and/or radiologically active, in which case the disease is considered relapsing-remitting. RR phenotype disease can also be either stable or can show clinical and/or radiological activity. The progressive disease phenotype can have four possible sub-classifications based on the presence of disease activity and/or progression:

- active, with progression (ie. the patient has had a relapse within the pre-defined time frame, also experiences gradually worsening symptoms),

- active, without progression (ie. the patient has had a relapse within a previously specified timeframe, but has no gradually worsening symptoms)

- not active, with progression (ie. no relapse has occurred, but has continuously worsening symptoms),

- not active, without progression (stable disease).

Figure 4.: Phenotypic classification of MS.

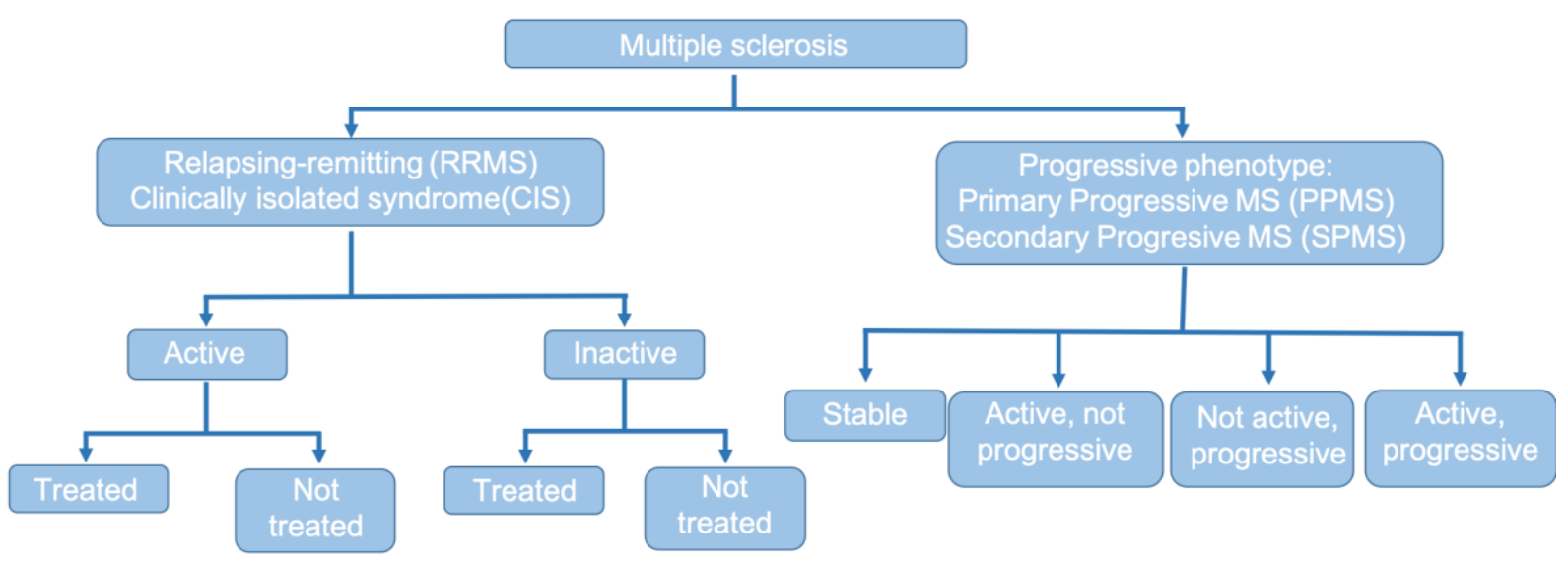

\section{1. 4. Definition of secondary progressive MS}

In the past decades, the Expanded Disability Status Scale (EDSS) was the sole and pivotal measurement system on which the disease subtype and clinical course classification relied [17]. There was no clear-cut margin defined, however, where the relapsing-remitting phase ended and secondary progressive phase (most commonly still with relapses) began. There was a significant, roughly 2 EDSS point wide overlap in the possible scores of the two disease courses, as the EDSS score of RR patients traditionally ranged from 0 to 5.5, whereas it resided between 3.5 and 10.0 for SP patients. Therefore all patients who previously fell into the 
relapsing-remitting disease type were re-evaluated for secondary progressive disease course using the objective, 3-strata criteria proposed by Lorscheider et al. for defining secondary progressive MS [24].

\section{III.2. Factors predicting and influencing the health-related quality of life of MS patients}

\section{2. 1. Patients}

A total of 322 patients have been enrolled from the MS outpatient clinics of the Department of Neurology of the University of Szeged, the Jahn Ferenc Dél-Pest Hospital of Budapest, and the Markhot Ferenc Teaching Hospital of Eger. For patients from Eger and Budapest, the patient data (sociodemographic, clinical, and psychological) were obtained from the patient documentation supplied by the patient's treating physician. Data of patients from Szeged were acquired from the multiple sclerosis registry of the Department of Neurology of the University of Szeged [54].

Inclusion criteria in the study were:

a) Informed consent from the patients.

b) A definitive diagnosis of MS according to the revised McDonald criteria of 2011.

c) Relapsing-remitting (not active, treated patients) or clinically isolated syndrome (not active, not treated) disease course.

d) At least 18 years of age.

f) The native language of the patient had to be Hungarian.

g) For the patient to be in remission, and not to have received steroid therapy for at least 30 days previous to the evaluation.

h) an EDSS score ranging from 0 to 6.5 .

Exclusion criteria were:

a) Any substance use, which may interfere with cognitive abilities or psychometric testing.

b) History of or ongoing chronic alcoholism or drug abuse.

c) Any concomitant or previous physical or psychiatric disorder other than MS that could affect the results of cognitive and/or psychiatric testing, or could affect the patients' mood with the exception of depression. 
d) Any neurological condition other than MS, that could have an impact on the result of the administered psychometric tests.

e) Progressive disease course.

The study was approved by the Ethics Committee of the Faculty of Medicine, University of Szeged (207/2015-SZTE). All participants gave their written informed consent in accordance with the Declaration of Helsinki.

\section{2. 2. Methods}

\section{2. 2. 1. Physical status}

The physical status and the level of disability of the patients was measured by the EDSS score [17], which was always determined by the patient's respective neurologist specialist at the time of enrollment.

\section{2. 2. 2. Psychopathological symptoms}

For the evaluation of psychopathological symptoms three, self-administered questionnaires were used; the BICAMS, the FIS, and the BDI tests were filled out by the patients.

\section{2. 2. 3. Cognitive status, cognitive impairment}

The cognitive status of the patients, the presence of cognitive impairment was measured by the administration of the Brief International Cognitive Assessment for MS (BICAMS) test validated to Hungarian language [47]. The BICAMS battery was designed to be a screening tool for the detection of cognitive impairment in MS patients. It can be rapidly administered (10-15 minutes), requires virtually no resources (other than the test subject, an examiner, pen, paper, and a stopwatch), yet encompasses very high sensitivity and specificity. It consists of 3 parts, which evaluate the information processing speed, the verbal memory and the visual memory of the subject; the orally administered version of the Symbol Digit Modalities Test (SDMT), the first 5 immediate recall trials of the California Verbal Learning Test-II (CVLTII), and the first 3 immediate recall trials of the Brief Visuospatial Memory Test-Revised (BVMT-R) [56]. A patient was considered to suffer from cognitive impairment if he/she had abnormal scores on at least one of the subtests, a criterion proposed by Dusankova et al. in 2012 [57]. A score was considered to be abnormal on a given test if it fell outside the predefined normal values of said test. 


\section{2. 2. 4. Mood}

For the evaluation of the patients' mood, the Hungarian validated version of the Beck Depression Inventory II (BDI II) was used [58]. The BDI is one of the most extensively utilized psychometric tests for the assessment of the presence of depression. It consists of 21 questions, exploring a patient's somatic, affective, and cognitive problems. Each question is self-rated, has 4 possible answers. The first answer is worth 0 points thus indicates no problem, while the last answer is worth 3 points, indicating a serious problem for the subject. In our study the internationally accepted standard cutoff values were used, a patient was considered to have depressive symptoms if scored at least 13 points.

\section{2. 2. 5. Fatigue}

For the evaluation of fatigue, the Hungarian version [59] of the Fatigue Impact Scale (FIS) was used. The questionnaire is self-administered, encompasses 40 questions, each of which is scored by the patient from 0 (representing no problem for the patient) to 4 (indicating a severe problem), making up a final scoring scale of 0-160 points. The form consists of three distinctly different internal subscales, which measure the cognitive, social, and physical dimensions of fatigue. The higher a subject scores the higher the patient feels to be burdened by fatigue. As a consensual cutoff value for this questionnaire does not exist, in our study a patient was classified to be significantly burdened by fatigue after scoring at least 40 points.

\section{2. 2. 6. Health-Related Quality of Life}

For the evaluation of the patients' HRQoL, we used the Hungarian version [60] of the Multiple Sclerosis Quality of Life-54 (MSQoL-54) questionnaire, which is the modified, expanded version of the SF-36 survey specifically tailored for the measurement of the HRQoL of MS patients. Its basis, the SF-36 comprises 36 questions that reflect on 8 domains of health; general health, mental health, physical functioning, physical role, emotional role, social functioning, pain, and vitality [61-66]. The SF-36 has proven to be a reliable and valid item for the measurement of health-related quality of life of individuals with diverse chronic health conditions. Its has been validated in several languages and is one of the most frequently used non-disease specific test in the world for the measurement of HRQoL. The MSQoL-54 form is made up of the 36 general questions found in the SF-36 survey, with the addition of 18 additional questions that are specific for MS. The MSQoL-54 questionnaire is extremely comprehensive, encompasses 12 distinct internal subscales, 2 summary scores, and 2 singleitem measures, which are highly sensitive to problems presenting in patients with MS. 


\section{2. 3. Statistical analysis}

To determine the different variables' level of influence on the HRQoL of the patients we used the model-free partial least squares regression (PLS) model. This was due to the fact that the commonly utilized multivariate regression model's results can be unreliable in circumstances when the examined predictors are not independent of each other. This was particularly true in our study as several of the examined predictors are intertwined and influence each other. In contrast to the regular multivariate regression model PLS successively extracts latent variables from the dependent variables and the predictors in such a way that covariance between the factors and loadings is maximized [67]. With this approach, PLS reduces the dimensionality of the data by providing a weighted linear combination of $X$ variables to form orthogonal components that predict the dependent variable. We analyzed the predictive values of the scores of the BICAMS, FIS, and BDI tests, as well as the patients' sex, level of education, age, disease duration, and EDSS score (predictive factors) on the different subscales of the MSQoL-54 questionnaire (dependent variables). The statistical inference on the significance of the latent variable was carried out by permutation tests on the singular values of the decomposition $(5,000$ permutations). An assessed predictive factor was considered to have a meaningful impact on a given subscale of the MSQoL-54 survey if the given parameter's variable importance of projection (VIP) score was $\geq 1$ [68]. The VIP scores are non-binary, the greater the score, the more important a given variable is considered to be to the model. On the other hand variables with VIP scores lower than 1 have a lower impact, and are considered to be less important predictive factors, thus are usually good candidates to be omitted from the model. To evaluate any potential differences between the clinical and sociodemographic variables of the patients we used one-way ANOVA and Fisher's exact test. 


\section{Results}

\section{1. Fresh epidemiological data based on the novel phenotypic classification of MS and patient treatment status}

On the prevalence day, $420 \mathrm{MS}$ patients were identified in the surveyed area, 313 women, and 107 men (female-male ratio of 2.92:1). The average age for the whole cohort was 48.83 $( \pm 10.64)$ years, age at diagnosis $34.15( \pm 10.64)$ years, average disease duration was 14.57 $( \pm 10.59)$ years, average EDSS score was $2.8( \pm 2.44)$ points (for detailed information about our cohort see Table 1).

Since the last sampling of the same area $30 \mathrm{MS}$ patients had died, 28 had moved away from the area, meanwhile, 108 new cases were discovered. We found the crude prevalence for the whole cohort to be 105.3/100,000, 56.5/100,000 for men, and 149.3/100,000 for women, respectively. The standardized prevalence for the whole cohort was 101.8/100,000. Age- and sex-adjusted, standardized prevalence was 53.9/100,000 for men and 144.8/100,000 for women (Table 2). Using the classical disease course definitions 12 patients $(2.86 \%)$ were identified with CIS, 30 (7.14\%) with primary progressive (PP), 102 (24.29\%) with secondary progressive (SP), and $276(65.71 \%)$ with relapsing-remitting (RR) disease type. There was no difference between the sexes regarding the distribution of disease courses $(\mathrm{p}=0.166)$. Based on the novel phenotypic classification $68.57 \%$ (288 patients) of our cohort was identified with a RR phenotype disease, of which 12 patients $(4.16 \%)$ had only a single attack, thus were classified as CIS, meanwhile progressive disease was observed in 132 patients (31.43\%). After stratification by phenotype and treatment status from the 276 R-R patients 28 (9.82\%) subject's disease showed activity despite being treated (active - treated arm, A-T), 15 (5.20\%) persons received no treatment despite showing disease activity (active - not treated arm, A-NT), in the case of the vast majority of R-R patients (202 persons, 70.13\%) no disease activity was seen whilst being treated (not active - treated arm, NA-T). A total of 43 people $(14.93 \%)$ were free of disease activity without a DMT (not active - not treated arm, NA-NT), of whom 20 patients had isolated optic neuritis, but with additional diagnostic measures according to the latest McDonald criteria the diagnosis of definite MS could be made. Their average disease duration, age, and age at diagnosis were $15.19( \pm 7.52), 50.28( \pm 14.57)$, and $34.77( \pm 10.82)$ years, had a mean EDSS score of $0.95( \pm 1.08)$ points (Tables 1 and 3$)$. 
Among patients with a progressive phenotype disease activity could be established in 31 patients; 23 persons' $(17.42 \%)$ disease showed progression adjacent to activity (active progressive arm, A-P), whilst progression wasn't seen despite disease activity in 8 patients $(6.06 \%$, active - not progressive arm, A-NP). A total of 53 (40.15\%) patients were identified who showed no progression nor disease activity (not active - not progressive arm, NA-NP), while no activity, but progression was seen in 48 (36.36\%) (not active - progressive arm, NAP) subjects. There was no difference in distribution in-between sexes $(p=0.258)$ regarding disease phenotypes (data not shown).

The overwhelming majority $(341,81.19 \%)$ of our patients had received some kind of immunomodulatory treatment for MS at any given time during their disease course. At the time of sampling 312 patients (74.28\%); 230 (79.86\%) patients with an RR disease type and 72 patients $(54.54 \%)$ with progressive disease type were treated with a DMT. A total of 178 patients received platform therapies, and 134 were prescribed a HADMT. As a first choice, 307 patients received platform drugs and 34 patients were started on a HADMT from the beginning of their treatment (Table 4).

Of the $28 \mathrm{RR}$ patients in the A-T arm, 10 patients were treated with platform drugs, 18 with HADMTs. Four patients have refused to change and escalate treatment, 2 patients have commenced a DMT less than 1 month before the prevalence day, hence their disease was still considered active and 22 patients have been switched to another DMT within 3 months of the prevalence day (ie. on their next visit). Of the 15 patients in the A-NT arm 3 patients began treatment shortly after the prevalence day, 1 patient has given birth not much prior to the prevalence day and has restarted treatment later, the rest, 11 patients either refused treatment or were unable to be treated because of compliance issues.

Roughly half (20 patients) of the 43 patients in the NA-NT group suffered solely 1 attack, comprising of optical neuritis and thus were closely observed and given no treatment yet. One patient was started on a DMT shortly after the prevalence day, meanwhile, 5 patients have been previously treated with platform therapies, but treatment cessation was necessary due to various reasons ( 2 patients became pregnant, 1 patient developed a malignant disease, 1 patient asked for the discontinuation of treatment, and 1 patient was unfit to be treated due to compliance issues).

The use of platform drugs was more common among RR patients (134 patients on platform vs. 68 on HADMT) than it was among patients with progressive disease (34 patients on platform 
vs. 48 on HADMT). The majority of our patients (134) were using their first choice of DMTs, treatment change was necessary once, twice, and thrice in the case of 103, 50, and 23 patients, respectively. Four and 5 treatment change was necessary with 1-1 patient (Table 4).

\section{2. Factors predicting and influencing the health-related quality of life of MS patients}

Of the 322 patients enrolled 102 (31.6\%) were men, and 220 (68.4\%) were women, the mean age of the examined population was $43( \pm 11.90)$ years, mean disease duration was $12.5( \pm 8.0)$ years. A little more than half of our cohort had received more than 12 years of education (171, $53.1 \%$ ), while 151 patients (46.9\%) were educated for 12 or fewer years. The average EDSS score of the patients was $1.95 \pm 1.60$ (range: $0-6.5$ ) points. Depression, fatigue, and cognitive impairment was identified in 87 (27.0\%), 168 (52.2\%), and 164 (50.9\%) patients, respectively. When the sexes were compared no difference was found in any of the aforementioned attributes, except for the prevalence of cognitive impairment, which was present in more men, than women (65 patients, $63.7 \%$ for men vs. 99 patients, $45.0 \%$ for women $p<.002$ ) (for detailed information about our cohort see Table 5).

Every examined factor's level of impact was evaluated against all of the MSQoL-54 questionnaire's 14 subscales separately. The evaluation was conducted independently for the whole cohort, and separately for men and women as well.

When the cohort was evaluated as a whole of all the 13 examined factors fatigue in general and depression were found to be the most prominent influencers of one's health-related quality of life. Depression, overall fatigue, and its composites (only physical, social, and cognitive fatigue were evaluated in our study) were observed to have a ubiquitous and profound effect on all subscales of the HRQoL composite (VIP score > 1 for all measured factors on every subscale) (see Table 6).

In every domain, where both fatigue and depression had a significant effect, the two of them had a roughly similar share of the cumulative impact, except for the emotional well-being and overall quality of life subscales, where fatigue's impact was less pronounced compared to depression's level of influence. The rest of the evaluated factors not only did influence fewer subscales but also did it with a lower power of effect. Age and the patients' EDSS score had an impact on only 3 , and 2 of the measured subscales, meanwhile disease duration, the level of education, and the presence of cognitive impairment did not have a clinically meaningful effect on any of the evaluated subscales when the cohort was assessed as a whole. Age had negatively 
affected the sexual function, satisfaction with sexual function, and physical health dimensions. For both sexes, as expected, the EDSS score only influenced the physical domain of the patients' HRQoL, it was found to be a negative determinant for the physical health (weaker contributor for women than for men) and physical role limitations subscales.

When the sexes were compared differences were found between male and female patients on 10 domains. Of all the evaluated factors depression and total fatigue were the only variables to have a major impact on all of the 14 examined domains of HRQoL (VIP > 1) for both sexes. In the case of men, in addition to total fatigue and depression, at least one other determinant was found to have a negative effect on the HRQoL in 9 domains, while for women such was found only in two domains.

Even though total fatigue proved to be a ubiquitous predicting factor some of its subsets fell short of having a clinically meaningful effect on a few dimensions of HRQoL; cognitive fatigue did not reach the level of significance on the change in health, emotional well-being, overall quality of life, satisfaction with sexual function and social function subscales for men, while social fatigue did not reach the threshold on the physical health subscale for men, and on the sexual function scale for women. Physical fatigue on the other hand had a major negative impact on all 14 subscales for both male and female patients. Having a higher level (more than 12 years of) education had a statistically significant effect solely on men's social function subscale, male patients with more education felt that their social life was negatively affected by MS. Cognitive impairment was a negative contributor on the role limitation due to emotional problems, sexual functions, the overall quality of life, and the satisfaction with sexual functions domain for men, but not women. Furthermore, the EDSS score proved to be a negative influencing factor of the health perceptions subscale only for men. For women, age was an additional clinical predictor for the sexual functions and satisfaction with sexual function dimensions, while it influenced the physical health and change in health subscales for men. 


\section{Discussion}

\section{1. Fresh epidemiological data based on the novel phenotypic classification and treatment status}

In the surveyed region the male:female ratio proved to be 1:1.106 among the general population, while for MS patients it was 1:2.925. The standardized prevalence of MS was found to be $101.8 / 100,000,53.9 / 100,000$ for men, and $144.8 / 100,000$ for women, meanwhile cumulative incidence was 4.44/100,000, 2.44/100,000 for men and 6.25/100,000 for women. Based on our current findings and previous epidemiological data from the same region Hungary is still considered a medium-risk country for MS [69-74] from an international perspective. A most recently published retrospective study from Hungary [75] with the prevalence day set in 2015 has found a slightly higher prevalence; they reported a cumulative standardized of 127.2/100,000 (175.6 for women and 74.7/100,000 for men), found the male:female ratio to be 1:2.6, observed a cumulative incidence of 5.1/100,000 (7.1/100,000 for women, 3.1/100,000 for men). A plausible explanation for the difference between the results of the two studies may be the differences in patient selection and the quality of the data on which the studies were based. Our study extracted data from a specialized MS registry gathered data from a well defined geographical region, meanwhile, the other study used administrative nationwide data supplied by the National Health Insurance Fund and extracted data from the local integrated hospital IT system, which holds the risk of using suboptimal quality data and usually makes the gathering of detailed individual clinical information on patients impossible.

The previous examination of the same geographical location we surveyed in our current work the male:female ratio was found to be 1:2.75 and 1:3.08 in the MS population and 1:1.09 and 1:1.12 in the overall county population in 1999 and 2013, respectively [72, 76]. Our findings of the continuous rise in the prevalence and female:male ratio of MS are in accordance with both current international and national findings in the literature [28, 75, 77-79]. Even though many theories [78] have seen the light in the past decades trying to explain the tendency (most of which are valid for Hungary as well), no unequivocal explanation for this rise in prevalence has been born yet. The constantly decreasing amount of childbirth in Europe [80], the increasing rate and severity of obesity in the developed world [19], high tobacco consumption [79, 81], and the role of vitamin $\mathrm{D}$ have been all been suspected to play a role in the increasing prevalence of MS [79]. Another fact which, at least in part, may explain this increasing tendency is the incredible amount of technological advancements experienced in the past few decades in imaging, laboratory testings, and the continuous revisions and evolving of the diagnostic 
criteria of MS, all of which allow us to make the diagnosis of MS faster than ever, thus may result in a greater perceived incidence and prevalence.

Furthermore, several DMTs (all of which are completely reimbursed by the national healthcare provider, therefore are available countrywide to every MS patient) have hit the market since the last assessment of the same region. Many of the new drugs introduced is a HADMT, which satisfied an unmet medical need, namely with these drugs the appropriate treatment became possible for patients in whom disease stability was not achieved with the „old, platform” therapies. This expansion of the therapeutic palette made personalized, tailored to disease activity treatment available for MS patients. This change in therapeutic approach has resulted in the considerable prolongation of the relapsing-remitting phase, therefore caused a significant delay in conversion to secondary progressive phase even for patients with very high disease activity, thus an increased overall survival of the patients can be seen nowadays compared to the times before the DMT (especially HADMT) era. We hypothesize that all of the aforementioned factors may, at least in part, be responsible for the observed rise in both incidence and prevalence of MS.

We found the distribution of different the disease courses in our cohort to be $2.86 \%$ CIS, $7.14 \%$ PPMS, 24.29\% SPMS, $65.71 \%$ RRMS while employing the novel phenotype-based classification [7] most of the cohort could be classified into RR/CIS phenotype $(68.57 \%, 288$ patients), progressive disease type was seen in 132 patients $(31.43 \%)$. The distribution of the classical disease courses fell in line with recent data based on large registries from both low and high prevalence countries across Europe [82-90]. However, except for the Argentinian cohort, none of these studies have used the most recent McDonald criteria, furthermore not one of these studies have evaluated their population according to the novel phenotypic classification or have given data about the disease activity of their patients, they only supply information about the prevalence of MS and the distribution of the classical disease courses. Additionally, fresh epidemiological data from the same climate zone that Hungary belongs to is lacking in the literature, a need that our study addresses. Globally ours was the first study to have used the most recent diagnostic and classification criteria for MS and to have also given information about the disease activity of the patients.

Almost every eligible patient received a DMT on the prevalence day, $83.33 \%$ of the patients with a RR/CIS disease type and $62.1 \%$ of the patients with a progressive disease received treatment. In the RR/CIS group the bigger part of the patients used platform drugs $(62.6 \% \mathrm{vs}$. 
37.4\% HADMT), while the distribution of drugs was more equal in the progressive disease group (41.5\% platform vs. 58.5\% HADMT). Most of the RR/CIS patients were successfully treated with their current DMT, as the overwhelming majority of patients have shown no disease activity (87.8\%), their mean EDSS (1.39) was low compared to their relatively long mean disease duration (11.55 years). Compared to RR/CIS patients a bigger ratio of patients with a progressive disease type has commenced their treatment with platform therapies $(87.4 \%$ vs. $75.34 \%$ ). The main reason behind this is the fact, that until recently there were no drugs approved for primary progressive disease course, and all of the since approved treatments for PPMS are HADMTs. Furthermore, most of the patients now in the secondary progressive phase began their treatment at a time in the past when only platform drugs were available for the treatment of RR disease type and converted to the secondary progressive phase before the introduction of HADMTs. Also, the lack of treatment options to choose from is the reason why patients in the progressive group underwent significantly fewer treatment changes, than did patients in the RR group. On the other hand, the relatively high number of patients treated with a HADMT is the result of the recent approval of ocrelizumab for the treatment of primary progressive disease in Hungary. Similarly, up until the past few years an escalative therapeutic approach was used in the case of the vast majority of the cohort, as when we began to treat most of our patients HADMTs were either not available yet at all or were not reimbursed by the healthcare provider as the first choice of treatment; therefore, only a lateral change or escalation was possible, induction with a highly potent drug was not.

Prior to our study, virtually no data from the HADMT era was available regarding the detailed treatment status of MS patients except for data published from Finland in 2019, which is based on one of the largest MS registries in the world containing the information of 8722 MS patients. The ratio of the used platform therapies vs HADMTs in RR and progressive MS in our cohort was similar to that seen in the Finnish population. On the other hand compared to the Finnish population in our cohort significantly more patients received treatment in both the RR and progressive group $(83.33 \%$ and $62.1 \%$ for RR and progressive disease type in our cohort vs. $66.0 \%$ and $16.0 \%$ in the Finnish cohort, respectively). Also, the ratio of the used platform vs. HADMTs in our cohort falls in line with the ratio reported from the MSBase and Swedish MS registries [91]. Furthermore only ours, but none of the above-mentioned studies have given indepth information about the current and previous treatment status of the patients that was correlated with their disease activity and phenotype. 
The observed trend in the high usage of HADMTs over platform therapies is expected to be even more pronounced in the following years, especially among patients with progressive disease. One of the reasons for this anticipated rise is the fact, that in the upcoming years more patients are going to be classified with secondary progressive disease type due to the introduction of the new diagnostic criteria. Another reason is that every drug currently in the pipeline awaiting EMA and/or FDA approval is considered a HADMT, moreover, the new therapeutic guideline advocates against the classical escalative approach and advises treating every patient as soon as possible with a DMT matching the patient's disease activity. As this guideline will eventually spread and will be implemented into everyday practice patients with high disease activity, who were previously prescribed platform therapies will be likely treated with a HADMT in the future.

\section{2. Factors predicting and influencing the health-related quality of life of MS patients}

One of the very first publications regarding the HRQoL of MS patients comes from 1992, it showed that when the HRQoL of patients with several different autoimmune disorders were compared MS patients perceived their health-related quality of life to be the worst [6]. Since then various authors have explored different aspects that might influence MS patients' quality of life, the earliest studies have found a negative correlation between the physical state and the HRQoL of MS patients [92-94]. In subsequent years other, previously rarely evaluated psychopathological symptoms have been at the center quality of life research's attention, fatigue, depression, and cognitive impairment all have been linked to a worse HRQoL and altered vocational status [42, 49, 95-99] in PwMS. Also, the independent effect of psychopathological symptoms from the patients' physical state on the HRQoL was shown [100]. None of these studies have evaluated multiple influencing factors simultaneously, however. Ours was among the first studies to have assessed multiple potential (and already proven) predicting factor's effect on the HRQoL of MS patients. Compared to previous studies we used a large, young, homogenous population of RR/CIS MS patients with low EDDS score and good physical condition, to minimize the distorting effect of several debilitating comorbidities, which burden the elderly and thus might significantly influence one's HRQoL. We simultaneously have examined all the major potential predictors of HRQoL of an MS patient; clinical disability (measured by the EDSS score), disease duration, age, the patients' sex, level of education, cognitive dysfunction, fatigue, and depression. 
Due to the diversity of the available tests that measure these predictors the results of the various studies usually are incomparable. In order to present comparable results for the evaluation of each factor, we employed the most predominantly used tests in the literature. While the EDSS score is not a perfect measure of disability (as it only takes into account the physical dimension of disability), it is omnipresent in the everyday clinical practice and scientific research as well, in the range our patients were enrolled (EDSS 0 to 6.5 points) the scale is nearly linear and shows an outstanding correlation with the patients' physical state [101]. As for the assessment of cognitive impairment the BICAMS battery was used, which has the potential to become the gold standard screening tool in assessing the cognitive capabilities of PwMS. Its components which are already in widespread use cover the most commonly affected domains in cognitively impaired PwMS [102, 103]. The same is true for depression and fatigue, they were assessed by one of the most ubiquitous screening tools in use, the FIS, and BDI questionnaires, respectively. The overall prevalence of psychopathological symptoms in our cohort $(52.2 \%$ for fatigue, $50.9 \%$ for cognitive impairment, and $27.0 \%$ for depression) are in line with data from the literature $[45,46,103]$, we also confirmed previous results about the observed difference in the prevalence of CI between women and men [47, 104].

In terms of the whole cohort, our results are mostly in agreement with recent studies, some differences are present, however. Similarly to other studies [42, 49, 95-97] we demonstrated that fatigue and depression have a ubiquitous, negative effect on all subscales of the MSQoL54, and also to be the most powerful determinants of HRQoL among the evaluated factors [49, 105-107]. The effect of other predictors was limited to solely one or two domains only; the EDSS score had a meaningful influence only on the physical aspects domain, while educational status only affected the social domain, to much surprise cognitive impairment, in general, seemed to be only a minor predictor for the self-perceived HRQoL for our MS patients. Accordingly, the current results in the literature on the topic is controversial, to say the least. Several results point in the direction that cognitive impairment is a primary determinant of the HRQoL of MS patients, while other studies relying on self-reported HRQoL measures did not find cognitive impairment to be a significant factor [39, 108-110]. The explanation behind the discrepancy of the results is multifactorial. One of the several possible reasons may be the fact that patients with severe enough cognitive disfunction are unaware of the decline in their cognitive skills, sometimes are burdened by other psychopathological symptoms - such as moria and euphoria - which distort their self-awareness even further, these patients are unable to give accurate descriptions about their own state, therefore their self-reports are unrealistic 
$[111,112]$. Another reason why CI had only a minor effect on our cohort's HRQoL might be explained by our patients' cognitive reserve capacity. The presence of higher levels of active and passive cognitive reserve capacity in PwMS has shown to be associated with higher levels of well-being, functional health, and lower levels of perceived disability in self-report surveys [113]. Yet another possible explanation might be the aforementioned connection between CI and the patients' vocational status, and the fact that CI seems to be mainly predictive of what PwMS are actually capable of doing, rather what they are able to report, thus these patients are unable to report their problems sufficiently [39, 49].

The literature about gender difference in MS is very scarce other than the well-known fact, that the prevalence of MS is much higher in women than men, and that male patients face a worse prognosis in general compared to women, especially in patients with late-onset MS [114-117]. Only very few studies have explored potential differences in the HRQoL of the sexes in MS, have found that men perceive their HRQoL significantly worse than women, especially in the physical, social functioning, and vitality domains $[116,118]$. None of these studies have explored the possibility, however, that there might be a difference in the very factors that influence the HRQoL of men, and women. We have demonstrated that age, physical disability, and CI had affected different aspects of men's and women's HRQoL, and also that having a higher education is a major determinant for men's, but not women's HRQoL. The physical status and age - two naturally linked aspects of one's physical decline over one's life span, even in healthy individuals - had influenced significantly more subscales for men, than they did for women, also their level of impact was more powerful in the case of men. One plausible explanation for this phenomenon might be rooted in how western societies nowadays still place great importance on one's physical stature, how ,value” much a member of society with a visible physical disability is perceived to lose. In spite of the huge IT boom in the past few years, the rapid advancement of IT technology, the emergence of jobs, that require no classical physical endurance and vitality, and even with the rapid spreading of home-office capable jobs (especially in the time of the recent Covid-19 outbreak) [119] western culture still places great value upon appearances, how a person's stature emanates efficiency and capability, expectations which are especially true for men. Thus living with a visible disability (which is not uncommon among MS patients) could invoke a sensation of failure to meet social standards, especially if someone was the wage-earner in a household and ceases to be able to fulfill this function due to a physical disability. This could be a more serious problem for men than women, as - even though the wage gap slowly, but is indeed closing [120] - in many western households 
men still are the breadwinners [121, 122]. As for why cognitive impairment had solely influenced the HRQoL of men, but not women may lie in the fact that brain atrophy is more pronounced - even in healthy - men [123], than it is in women, brain atrophy has been shown to be even more hastened by MS [124], increased atrophy has been associated with accelerated disability progression in PwMS [125]. Furthermore, cognitive impairment's prevalence is much higher in men, than women, and thus its impact might have reached a threshold where CI's burden becomes a significant factor for men, but not women [47]. Additionally cognitively impaired MS patients are more likely to become unemployed [39], the presence of CI has been linked to an increased number of divorces and self-reported decline in sexual functions among PwMS [126], which has been shown to further reduce the patients' HRQoL [127]. As mentioned above, patients with severe enough cognitive impairment are usually not able to characterize the exact problem present (and therefore these patients generally tend to score higher on self-report HRQoL surveys), but can, to an extent feel, that something has turned for the worse. This might be the basis of our finding, that cognitive impairment can have a negative impact on one's overall quality of life, without having a separate effect on the different subscales making up one's HRQoL. Having a high level of education had solely affected male patients' social function composite, with a level of influence matching fatigue's and depression's power on their respectively influenced composites. This is a somewhat expected result, as in western societies people with higher education tend to have better chances at attaining well-paying jobs, have better work hours than mechanical labourers do, also have better prospects and economic prosperity [128]. A sudden fall—may it be real or only perceived — from this higher social and economical state, can have a huge impact on one's HRQoL, especially for patients with a debilitating sickness, such as MS, which one can feel has absolutely no control over. This is particularly true for men who are still wage-earners in many societies. Information about sex's effect on the HRQoL of PwMS is virtually nonexistent in the literature, ours is among the first studies to show that different factors with different power influence different aspects of the HRQoL of male and female MS patients.

\section{Conclusion}

\section{1. Fresh epidemiological data based on the novel phenotypic classification and treatment status}

Our study is the first in the literature, that supplies epidemiological information about MS using the latest diagnostic guidelines and phenotypic classification system. Our findings of the rising prevalence and the female/male ratio shifting further towards women are in line with most 
recent reports, additionally, the observed stable incidence rate is also consistent with data found in the literature. In addition, ours is the second to the Finnish cohort [83] that gives information about the treatment status of the patients in addition to prevalence data and not solely the number of patients with different disease courses. Our work is, however, the first in the literature, that simultaneously gives detailed data about the physical status, treatment status, and disease course distribution of an MS cohort, does it using the most up to date classification and diagnostic criteria. Even though our sample size is relatively small in comparison to other epidemiological studies - predominantly originating from northern countries - our findings regarding the distribution of the disease courses are similar to those seen in a most recent report from Finland [83]. Epidemiological data from the Central European region based on the recent diagnostic and classification criteria were lacking for MS, which demand our study addressed.

\section{2. Factors predicting and influencing the health-related quality of life of MS patients}

Prior to our study all previous reports addressing the HRQoL of MS patients only focused on one or a very select few factors that might influence the patients' HRQoL. Ours was the first study in the literature that has examined the effect of many determinants simultaneously on several distinct aspects of one's HRQoL. Furthermore, our study is exceptional, as compared to other studies we have examined a large, young, homogenous population that was in good health despite the relatively long average disease duration, also other than the prevalence of cognitive impairment there was no difference between women and men in any attributes. Our findings thus cannot be attributed to the patients' bad physical state, cognitive decline, or the distorting effect of the psychopathological symptoms that are frequently prevalent in the late stages of MS. Our results regarding fatigue and depression corroborate other recent studies' findings [106, 129]; we too have found these two factors to be the main determining factors of MS patients HRQoL many years into their disease, not only in the short period of time following disease onset and diagnosis, as it was previously thought [130]. However, we have shown that several other factors in play have a significant influence on a number of different aspects of the patients' HRQoL. Furthermore, we have demonstrated that different factors with different power influence the HRQoL of men and women.

Our results urge a paradigm shift in the treatment of MS patients and invoke the need for the regular assessment of the aforementioned psychopathological symptoms with a multidimensional, personalized, quantitative approach on top of the regular neurological 
examination. The involvement of psychological and psychiatric specialists and teamwork for the proper management of MS patients is also called for. This is of exceptional importance, as many of the factors that we have shown to play a major role in determining the patients' HRQoL have been demonstrated to be ameliorated and managed by not only a psychopharmacological but by a psychotherapeutic approach as well [103, 107, 131]. 
Tables

Table 1.: Demographic and clinical characteristics of our cohort

\begin{tabular}{|c|c|c|c|c|c|c|c|c|c|c|c|c|c|c|c|c|c|c|}
\hline & \multicolumn{4}{|c|}{ Age } & \multicolumn{4}{|c|}{ Age at diagnosis } & \multicolumn{4}{|c|}{ Disease duration } & \multicolumn{3}{|c|}{ EDSS } & \multirow[b]{2}{*}{ SD } \\
\hline & & & Mean & Median & IQR & SD & Mean & Median & IQR & SD & Mean & Median & IQR & SD & Mean (range) & Median & IQR & \\
\hline \multicolumn{3}{|c|}{ Cohort $(420,100 \%)$} & 48,83 & 48,00 & 20,00 & 13,23 & 34,15 & 34,00 & 14,00 & 10,64 & 14,57 & 12,0 & 15,00 & 10,59 & $2,804(0-9.5)$ & 2,00 & 3,50 & 2,44 \\
\hline \multirow{7}{*}{$\begin{array}{c}\text { RR disease } \\
(288, \\
68.57 \%)\end{array}$} & \multirow{3}{*}{ Disease course } & $\mathrm{RR}+\mathrm{CIS}(288)$ & 44,42 & 44,00 & 17,00 & 11,55 & 32.58 & 32,00 & 14,00 & 9.933 & 11.73 & 10,0 & 11,00 & 8.372 & $1.375(0-3.5)$ & 1,50 & 2,00 & 1,08 \\
\hline & & CIS $(12 ; 4.16 \%)$ & 42,50 & 39,50 & 19,00 & 13,51 & 30,67 & 25,00 & 13,00 & 12,94 & 11,25 & 7,5 & 14,00 & 8,21 & $0,083(0-1.0)$ & 0,00 & 0,00 & 0,29 \\
\hline & & RR $(276 ; 95.84 \%)$ & 44,51 & 44,00 & 16,00 & 11,48 & 32,67 & 32,00 & 13,00 & 9,80 & 11,75 & 10,0 & 11,00 & 8,39 & $1,431(0-3.5)$ & 1,50 & 2,00 & 1,07 \\
\hline & \multirow{4}{*}{$\begin{array}{l}\text { Activity - treatment } \\
\text { status }\end{array}$} & A-T $(28 ; 9.72 \%)$ & 42,46 & 44,00 & 17,00 & 9,46 & 33,07 & 34,50 & 13,00 & 9,25 & 9,39 & 8,0 & 6,00 & 6,30 & $1,821(0-3.5)$ & 2,00 & 1,50 & 0,85 \\
\hline & & A-NT $(15 ; 5.2 \%)$ & 43,33 & 46,00 & 14,00 & 11,39 & 35,80 & 33,00 & 15,00 & 8,59 & 8,53 & 7,0 & 6,00 & 9,30 & $1,5(0-3.5)$ & 1,00 & 1,50 & 1,13 \\
\hline & & NA-T $(202 ; 70.13 \%)$ & 42,53 & 43,00 & 16,00 & 10,79 & 31,81 & 31,00 & 13,00 & 9,87 & 11,55 & 10,0 & 10,00 & 7,52 & $1,394(0-3.5)$ & 1,50 & 1,50 & 1,08 \\
\hline & & NA-NT $(43,14.93 \%)$ & 50,28 & 48,00 & 27,00 & 14,57 & 34,77 & 34,00 & 13,00 & 10,82 & 15,19 & 10,0 & 17,00 & 7,52 & $0,953(0-3.5)$ & 1,00 & 1,50 & 1,08 \\
\hline \multirow{7}{*}{$\begin{array}{c}\text { Progressive } \\
\text { disease } \\
(132, \\
31.43 \%)\end{array}$} & \multirow{3}{*}{ Disease course } & $\mathrm{PP}+\mathrm{SP}(132)$ & 58,44 & 60,00 & 15,00 & 11,48 & 37,58 & 37,00 & 16,00 & 11,33 & 20,77 & 21,0 & 19,00 & 12,20 & $5.92(3.0-9.5)$ & 6,00 & 1,90 & 1,46 \\
\hline & & PP (30; 22.73\%) & 58,53 & 60,50 & 13,00 & 10,24 & 48,33 & 48,00 & 14,00 & 9,73 & 10,17 & 8,0 & 11,00 & 8,11 & 5,617 (3.0-9.5) & 6,00 & 3,00 & 1,73 \\
\hline & & SP $(102 ; 77.27 \%)$ & 58,41 & 60,00 & 16,00 & 11,86 & 34,42 & 34,00 & 13,00 & 9,74 & 23,88 & 24,0 & 15,00 & 11,44 & $6,01(4.0-9.0)$ & 6,00 & 2,00 & 1,37 \\
\hline & \multirow{4}{*}{$\begin{array}{c}\text { Activity - treatment } \\
\text { status }\end{array}$} & A-NP $(8 ; 6.06 \%)$ & 49,38 & 47,50 & 17,00 & 8,99 & 35,13 & 37,00 & 22,00 & 13,23 & 14,25 & 14,5 & 21,00 & 10,08 & $5,313(3.5-8.5)$ & 5,00 & 2,40 & 1,65 \\
\hline & & A-P $(23 ; 17.42 \%)$ & 49,91 & 50,00 & 16,00 & 10,46 & 34,74 & 35,00 & 15,00 & 10,35 & 15,17 & 13,0 & 16,00 & 10,49 & $5,652(3.0-7.5)$ & 6,00 & 2,00 & 1,28 \\
\hline & & NA-P $(48 ; 36.36 \%)$ & 62,33 & 63,00 & 16,00 & 11,16 & 40,17 & 38,50 & 16,00 & 12,49 & 22,06 & 22,5 & 16,00 & 12,56 & $6,542(3.0-6.5)$ & 6,50 & 2,00 & 1,55 \\
\hline & & NA-NP $(53 ; 40.15 \%)$ & 59,98 & 62,00 & 17,00 & 10,05 & 36,85 & 36,00 & 20,00 & 10,11 & 23,00 & 24,0 & 21,00 & 12,04 & 5,566 (3.0-9.0) & 5,50 & 1,50 & 1,25 \\
\hline
\end{tabular}

Abbreviations: IQR, interquartile range; SD, standard deviation; RR, relapsing-remitting; CIS, clinically isolated syndrome; PP, primary progressive; SP, secondary progressive; A, active; NA, not active; T, treated; NT, not treated; P, progressive; NP, not progressive. Age and disease duration are given in years, EDSS score is given in points. 
Table 2.: Age-, and sex-specific crude and standardized prevalence of multiple sclerosis among men and women in Csongrád county on the prevalence day of $1^{\text {st }}$ January 2019.

\begin{tabular}{|c|c|c|c|c|c|c|}
\hline & \multicolumn{2}{|c|}{ Total } & \multicolumn{2}{|c|}{ Women } & \multicolumn{2}{|c|}{ Men } \\
\hline $\begin{array}{c}\text { Age group } \\
\text { (years) }\end{array}$ & Cases & Prevalence & Cases & Prevalence & Cases & Prevalence \\
\hline $0-14$ & 0 & 0,0 & 0 & 0,0 & 0 & 0,0 \\
\hline $15-19$ & 1 & 5,0 & 1 & 10,3 & 0 & 0,0 \\
\hline $20-24$ & 4 & 16,0 & 3 & 24,2 & 1 & 8,0 \\
\hline $25-29$ & 23 & 84,1 & 19 & 142,9 & 4 & 28,5 \\
\hline $30-34$ & 38 & 157,4 & 29 & 250,4 & 9 & 71,7 \\
\hline $35-39$ & 46 & 177,3 & 30 & 234,6 & 16 & 121,7 \\
\hline $40-44$ & 59 & 176,0 & 47 & 285,4 & 12 & 70,4 \\
\hline $45-49$ & 57 & 198,2 & 37 & 257,9 & 20 & 138,7 \\
\hline $50-54$ & 49 & 186,6 & 37 & 277,5 & 12 & 92,8 \\
\hline $55-59$ & 43 & 183,8 & 36 & 290,6 & 7 & 63,6 \\
\hline $60-64$ & 45 & 153,9 & 34 & 211,2 & 11 & 83,7 \\
\hline $65-69$ & 32 & 123,0 & 19 & 126,8 & 13 & 117,8 \\
\hline $70-74$ & 14 & 67,0 & 14 & 111,2 & 0 & 0,0 \\
\hline $75-79$ & 4 & 24,7 & 4 & 39,2 & 0 & 0,0 \\
\hline $80-84$ & 3 & 28,9 & 2 & 28,4 & 1 & 30,0 \\
\hline $85-$ & 2 & 23,3 & 1 & 16,2 & 1 & 41,1 \\
\hline Total & 420 & & 313 & & 107 & \\
\hline Crude* & & 105,3 & & 149,3 & & 56,5 \\
\hline $\begin{array}{c}\text { Age-sex } \\
\text { adjusted** }\end{array}$ & & 101,8 & & 144,8 & & 53,9 \\
\hline
\end{tabular}

Note: The average incidence of MS for the examined period was 4.44/100,000 in total, was 6.25/100,000 for women, and 2.44/100,000 for men, respectively. ${ }^{*}$ Crude prevalence per 100,000 persons. ${ }^{* *}$ Standardized prevalence per 100,000 persons, in the direct standardization the 2013 European standard population was used as a reference population. 
Table 3.: The distribution of the patients across the EDSS scale stratified by disease type and activity.

EDSS

\begin{tabular}{llllllllllllllllllllll}
0 & 1 & 1,5 & 2 & 2,5 & 3 & 3,5 & 4 & 4,5 & 5 & 5,5 & 6 & 6,5 & 7 & 7,5 & 8 & 8,5 & 9 & 9,5 \\
\hline
\end{tabular}

\begin{tabular}{|c|c|c|c|c|c|c|c|c|c|c|c|c|c|c|c|c|c|c|c|c|c|}
\hline \multirow{6}{*}{$\begin{array}{c}\text { RR disease } \\
(288, \\
68.57 \%)\end{array}$} & \multirow{2}{*}{ Disease course } & CIS $(12 ; 4.16 \%)$ & 11 & 1 & 0 & 0 & 0 & 0 & 0 & 0 & 0 & 0 & 0 & 0 & 0 & 0 & 0 & 0 & 0 & 0 & 0 \\
\hline & & RR $(276 ; 95.84 \%)$ & 71 & 52 & 35 & 58 & 22 & 27 & 11 & 0 & 0 & 0 & 0 & 0 & 0 & 0 & 0 & 0 & 0 & 0 & 0 \\
\hline & \multirow{4}{*}{$\begin{array}{c}\text { Activity - treatment } \\
\text { status }\end{array}$} & A-T $(28 ; 9.72 \%)$ & 1 & 8 & 4 & 7 & 4 & 2 & 2 & 0 & 0 & 0 & 0 & 0 & 0 & 0 & 0 & 0 & 0 & 0 & 0 \\
\hline & & A-NT $(15 ; 5.2 \%)$ & 3 & 5 & 1 & 2 & 1 & 2 & 1 & 0 & 0 & 0 & 0 & 0 & 0 & 0 & 0 & 0 & 0 & 0 & 0 \\
\hline & & NA-T $(202 ; 70.13 \%)$ & 58 & 31 & 26 & 46 & 14 & 20 & 7 & 0 & 0 & 0 & 0 & 0 & 0 & 0 & 0 & 0 & 0 & 0 & 0 \\
\hline & & NA-NT $(43,14.93 \%)$ & 20 & 9 & 4 & 3 & 3 & 3 & 1 & 0 & 0 & 0 & 0 & 0 & 0 & 0 & 0 & 0 & 0 & 0 & 0 \\
\hline \multirow{6}{*}{$\begin{array}{c}\text { Progressive } \\
\text { disease } \\
(132, \\
31.43 \%)\end{array}$} & \multirow{2}{*}{ Disease course } & PP (30; 22.73\%) & 0 & 0 & 0 & 0 & 0 & 0 & 0 & 18 & 3 & 9 & 12 & 13 & 20 & 9 & 8 & 3 & 3 & 4 & 0 \\
\hline & & SP $(102 ; 77.27 \%)$ & 0 & 0 & 0 & 0 & 0 & 4 & 4 & 0 & 1 & 0 & 4 & 6 & 5 & 1 & 2 & 2 & 0 & 0 & 1 \\
\hline & \multirow{4}{*}{$\begin{array}{c}\text { Activity - treatment } \\
\text { status }\end{array}$} & A-NP $(8 ; 6.06 \%)$ & 0 & 0 & 0 & 0 & 0 & 0 & 1 & 2 & 0 & 2 & 0 & 1 & 1 & 0 & 0 & 0 & 1 & 0 & 0 \\
\hline & & A-P $(23 ; 17.42 \%)$ & 0 & 0 & 0 & 0 & 0 & 1 & 1 & 3 & 1 & 1 & 4 & 2 & 5 & 4 & 1 & 0 & 0 & 0 & 0 \\
\hline & & NA-P $(48 ; 36.36 \%)$ & 0 & 0 & 0 & 0 & 0 & 1 & 2 & 9 & 2 & 5 & 9 & 8 & 11 & 2 & 2 & 1 & 0 & 1 & 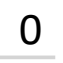 \\
\hline & & NA-NP $(53 ; 40.15 \%)$ & 0 & 0 & 0 & 0 & 0 & 2 & 0 & 4 & 1 & 1 & 3 & 8 & 8 & 4 & 7 & 4 & 2 & 3 & 1 \\
\hline
\end{tabular}

Note: Each column indicates the number of patients with that exact EDSS score in a given disease type or disease activity subgroup. Abbreviations: A-NP, active-not progressive (patient showing disease without progression); A-NT, active-not treated (patients showing disease activity without receiving treatment); A-P, active-progressive (patient showing disease and progression at the same time); A-T, active-treated (patients showing disease activity despite receiving treatment); CIS, clinically isolated syndrome; NA-NP, not active-not progressive (patient not showing disease activity nor progression); NA-NT, not active-not treated (patient not showing disease activity without receiving treatment); NA$\mathrm{P}$, not active- progressive (patient not showing disease activity while showing progression); NA-T, not active-treated (patient not showing disease activity while receiving treatment); PP, primary progressive disease; R-R, relapsing-remitting; SP, secondary progressive disease. 
Table 4.: Present and past treatment status, the number of necessary treatment changes in our patients stratified by disease type and disease activity.

\begin{tabular}{|c|c|c|c|c|c|c|c|c|c|c|c|c|c|c|c|c|c|}
\hline & & & \multicolumn{2}{|c|}{ Treated ever } & \multicolumn{3}{|c|}{ Treated now } & \multicolumn{2}{|c|}{ Started on HADMT } & \multicolumn{2}{|c|}{ Started on platform } & \multicolumn{6}{|c|}{ Number of DMT changes } \\
\hline & & & No & Yes & No & Platform & HADMT & No & Yes & No & Yes & 0 & 1 & 2 & 3 & 4 & 5 \\
\hline \multirow{5}{*}{$\begin{array}{c}\text { RR disease } \\
(288,68.57 \%)\end{array}$} & CIS $(12 ; 4.16 \%)$ & NA-NT & 12 & 0 & 12 & 0 & 0 & 12 & 0 & 12 & 0 & 0 & 0 & 0 & 0 & 0 & 0 \\
\hline & \multirow{4}{*}{ RR (276; 95.84\%) } & A-T & 0 & 28 & 0 & 10 & 18 & 27 & 1 & 1 & 27 & 7 & 11 & 8 & 1 & 0 & 1 \\
\hline & & A-NT & 12 & 3 & 15 & 0 & 0 & 15 & 0 & 12 & 3 & 1 & 2 & 0 & 0 & 0 & 0 \\
\hline & & NA-T & 0 & 202 & 0 & 134 & 68 & 182 & 20 & 20 & 182 & 96 & 66 & 26 & 13 & 1 & 0 \\
\hline & & NA-NT & 26 & 5 & 31 & 0 & 0 & 31 & 0 & 26 & 5 & 3 & 1 & 1 & 0 & 0 & 0 \\
\hline \multirow{8}{*}{$\begin{array}{c}\text { Progressive } \\
\text { disease } \\
(132,31.43 \%)\end{array}$} & \multirow{4}{*}{ PP (30; 22.73\%) } & A-NP & 0 & 2 & 0 & 1 & 1 & 1 & 1 & 1 & 1 & 1 & 0 & 0 & 1 & 0 & 0 \\
\hline & & A-P & 3 & 3 & 3 & 0 & 3 & 4 & 2 & 5 & 1 & 2 & 0 & 1 & 0 & 0 & 0 \\
\hline & & NA-NP & 2 & 5 & 2 & 1 & 4 & 4 & 3 & 5 & 2 & 4 & 0 & 1 & 0 & 0 & 0 \\
\hline & & NA-P & 7 & 8 & 8 & 0 & 7 & 9 & 6 & 13 & 2 & 7 & 1 & 0 & 0 & 0 & 0 \\
\hline & \multirow{4}{*}{ SP $(102 ; 77.27 \%)$} & A-NP & 0 & 6 & 1 & 2 & 3 & 5 & 1 & 1 & 5 & 1 & 3 & 2 & 0 & 0 & 0 \\
\hline & & A-P & 1 & 16 & 5 & 3 & 9 & 17 & 0 & 1 & 16 & 4 & 5 & 5 & 2 & 0 & 0 \\
\hline & & NA-NP & 4 & 42 & 7 & 22 & 17 & 46 & 0 & 4 & 42 & 15 & 15 & 6 & 6 & 0 & 0 \\
\hline & & NA-P & 12 & 21 & 24 & 5 & 4 & 33 & 0 & 12 & 21 & 10 & 5 & 5 & 1 & 0 & 0 \\
\hline
\end{tabular}

Note: Columns one and two show how many of our patients in a subgroup have ever been treated during some point in their disease, also the potency of the used DMT for patients who received treatment on the prevalence day. Columns three and four show how many patients in each subgroup have started their treatment with low or high potency drugs. Most of the still treated R-R patients started their treatment with platform drugs, escalation to a HADMT was necessary only in 48 cases. On the contrary, a higher fraction of patients with a progressive disease started their treatment with a HADMT. Furthermore, a bigger ratio of PP and SP patients switched onto a HADMT after being started on platform drugs, than did patients from the RR disease type group. Column five shows the number of instances therapy change was necessary for patients in a given subgroup. Abbreviations: A-NP, active-not progressive (patient showing disease without progression); A-NT, active-not treated (patients showing disease activity without receiving treatment); A-P, active-progressive (patient showing disease and progression at the same time); A-T, activetreated (patients showing disease activity despite receiving treatment); CIS, clinically isolated syndrome; HADMT, highly active disease-modifying treatment-fingolimod, natalizumab, ocrelizumab, cladribine, alemtuzumab, and mitoxantrone; NA-NP, not active-not progressive (patient not showing disease activity nor progression); NA-NT, not active-not treated (patient not showing disease activity without receiving treatment); NA$\mathrm{P}$, not active-progressive (patient not showing disease activity while showing progression); NA-T, not active-treated (patient not showing disease activity while receiving treatment); Platform, platform therapies-interferons, glatiramer acetate, dimethyl fumarate, and teriflunomide; PP, primary progressive disease; R-R, relapsing-remitting; SP, secondary progressive disease. 
Table 5.: The sociodemographic and clinical characteristics of our cohort with the comparison of the sexes.

\begin{tabular}{|c|c|c|c|c|}
\hline & Whole cohort & Women & Men & Difference (p) \\
\hline Age at enrollment (min, max, median, IQR, SD) & $42.96(21.0,69.0,42.0,18.0,11.88)$ & $43.71(21.0,69.0,44.0,19.011 .72)$ & $41.34(22.0,68.0,39.5,16.0,12.10)$ & 0.096 \\
\hline Disease duration (min, max, median, IQR, SD) & $12.48(1.0,45.0,11.0,11.0,7.95)$ & $12.48(1.0,41.0,11.0,11.0,7.57)$ & $12.48(1.0,45.0,10.5,12.0,8.74)$ & 0.997 \\
\hline Patients with cognitive impairment $(\%)$ & $164(50.9)$ & $99(45.0)$ & $65(63.7)$ & 0.002 \\
\hline Patients with depressive symptoms (\%) & $87(27.0)$ & $62(28.2)$ & $25(24.5)$ & 0.590 \\
\hline Patients burdened by fatigue $(\%)$ & $168(52.2)$ & $118(53.6)$ & $50(49.0)$ & 0.473 \\
\hline EDSS (min, max, median, IQR, SD) [points] & $1.95(0,6.5,2.0,2.01 .57)$ & $1.85(0,6.5,1.5,1.5,1.48)$ & $2.16(0,6.5,2.0,2.0,1.73)$ & 0.097 \\
\hline No. of patients with high education (\%) & $171(53.1)$ & $115(52.3)$ & $56(54.9)$ & 0.661 \\
\hline No. of patients enrolled & 322 & 220 & 102 & $\mathrm{n} / \mathrm{a}$ \\
\hline
\end{tabular}

Note: All of the variables' values are shown in mean, disease duration, and age are given in years. Abbreviations: EDSS, Expanded Disability Status Scale Score; max, maximum; min, minimum; IQR, interquartile range; SD, standard deviation. 
Table 6.: Variable importance in projection (VIP) scores of the different factors contributing to the HRQoL of our patients measured by the different subscales of MSQoL-54 questionnaire in the whole cohort and the different sexes.

\begin{tabular}{|c|c|c|c|c|c|c|c|c|c|c|c|c|c|}
\hline & Sex & Age & $\begin{array}{l}\text { Disease } \\
\text { duration }\end{array}$ & EDSS & Education & $\begin{array}{c}\text { BVMT-R } \\
\text { score }\end{array}$ & $\begin{array}{c}\text { SDMT } \\
\text { score }\end{array}$ & $\begin{array}{c}\text { CVLT-II } \\
\text { score }\end{array}$ & Total fatigue & $\begin{array}{c}\text { Cognitive } \\
\text { fatigue }\end{array}$ & $\begin{array}{c}\text { Physical } \\
\text { fatigue }\end{array}$ & $\begin{array}{r}\text { Social } \\
\text { fatigue }\end{array}$ & Depression \\
\hline \multirow{3}{*}{ Change in health } & Overall & 0.943 & 0.466 & 0.915 & 0.530 & 0.414 & 0.438 & 0.242 & 1.575 & 1.452 & 1.507 & 1.320 & 1.321 \\
\hline & Women & 0.747 & 0.375 & 0.885 & 0.475 & 0.385 & 0.515 & 0.265 & 1.550 & 1.521 & 1.486 & 1.236 & 1.164 \\
\hline & Men & 1.412 & 0.657 & 0.869 & 0.606 & 0.393 & 0.116 & 0.117 & 1.304 & 0.942 & 1.240 & 1.294 & 1.572 \\
\hline \multirow{3}{*}{ Cognitive function } & Overall & 0.756 & 0.534 & 0.650 & 0.512 & 0.562 & 0.665 & 0.719 & 1.553 & 1.431 & 1.430 & 1.378 & 1.330 \\
\hline & Women & 0.674 & 0.532 & 0.646 & 0.432 & 0.663 & 0.765 & 0.653 & 1.475 & 1.342 & 1.332 & 1.369 & 1.257 \\
\hline & Men & 0.822 & 0.477 & 0.623 & 0.624 & 0.309 & 0.488 & 0.795 & 1.494 & 1.420 & 1.442 & 1.165 & 1.291 \\
\hline \multirow{3}{*}{ Emotional well-being } & Overall & 0.681 & 0.531 & 0.579 & 0.453 & 0.550 & 0.546 & 0.708 & 1.449 & 1.269 & 1.384 & 1.271 & 1.836 \\
\hline & Women & 0.698 & 0.537 & 0.615 & 0.529 & 0.516 & 0.422 & 0.644 & 1.382 & 1.314 & 1.349 & 1.105 & 1.741 \\
\hline & Men & 0.540 & 0.452 & 0.451 & 0.205 & 0.588 & 0.807 & 0.752 & 1.376 & 0.962 & 1.246 & 1.477 & 1.773 \\
\hline \multirow{3}{*}{ Energy } & Overall & 0.693 & 0.478 & 0.677 & 0.321 & 0.263 & 0.337 & 0.248 & 1.636 & 1.598 & 1.517 & 1.365 & 1.446 \\
\hline & Women & 0.667 & 0.416 & 0.702 & 0.379 & 0.187 & 0.309 & 0.145 & 1.570 & 1.613 & 1.457 & 1.260 & 1.348 \\
\hline & Men & 0.651 & 0.546 & 0.569 & 0.139 & 0.437 & 0.416 & 0.445 & 1.550 & 1.328 & 1.434 & 1.416 & 1.465 \\
\hline \multirow{3}{*}{ Health distress } & Overall & 0.494 & 0.287 & 0.767 & 0.321 & 0.485 & 0.399 & 0.553 & 1.580 & 1.398 & 1.569 & 1.296 & 1.637 \\
\hline & Women & 0.460 & 0.275 & 0.719 & 0.332 & 0.488 & 0.348 & 0.604 & 1.506 & 1.417 & 1.483 & 1.198 & 1.565 \\
\hline & Men & 0.547 & 0.286 & 0.787 & 0.247 & 0.369 & 0.445 & 0.327 & 1.536 & 1.136 & 1.566 & 1.361 & 1.595 \\
\hline \multirow{3}{*}{ Health perception } & Overall & 0.870 & 0.502 & 0.976 & 0.469 & 0.413 & 0.465 & 0.269 & 1.533 & 1.429 & 1.510 & 1.215 & 1.467 \\
\hline & Women & 0.856 & 0.436 & 0.820 & 0.470 & 0.357 & 0.432 & 0.277 & 1.498 & 1.500 & 1.454 & 1.146 & 1.368 \\
\hline & Men & 0.858 & 0.579 & 1.128 & 0.418 & 0.373 & 0.336 & 0.179 & 1.424 & 1.094 & 1.463 & 1.212 & 1.538 \\
\hline \multirow{3}{*}{ Overall quality of life } & Overall & 0.472 & 0.228 & 0.850 & 0.518 & 0.665 & 0.752 & 0.669 & 1.439 & 1.284 & 1.395 & 1.217 & 1.761 \\
\hline & Women & 0.487 & 0.108 & 0.844 & 0.551 & 0.458 & 0.522 & 0.575 & 1.427 & 1.347 & 1.359 & 1.194 & 1.673 \\
\hline & Men & 0.411 & 0.519 & 0.711 & 0.333 & 1.093 & 1.207 & 0.799 & 1.183 & 0.866 & 1.237 & 1.015 & 1.716 \\
\hline \multirow{3}{*}{ Pain } & Overall & 0.915 & 0.659 & 0.814 & 0.439 & 0.204 & 0.312 & 0.197 & 1.545 & 1.450 & 1.450 & 1.313 & 1.538 \\
\hline & Women & 0.791 & 0.471 & 0.686 & 0.522 & 0.233 & 0.422 & 0.186 & 1.495 & 1.439 & 1.411 & 1.246 & 1.515 \\
\hline & Men & 1.029 & 0.928 & 0.996 & 0.195 & 0.189 & 0.172 & 0.213 & 1.435 & 1.265 & 1.327 & 1.282 & 1.370 \\
\hline \multirow{3}{*}{ Physical health } & Overall & 1.066 & 0.778 & 1.294 & 0.396 & 0.448 & 0.451 & 0.296 & 1.484 & 1,470 & 1.496 & 1.059 & 1.100 \\
\hline & Women & 0.964 & 0.690 & 1.149 & 0.438 & 0.368 & 0.492 & 0.315 & 1.451 & 1.485 & 1.429 & 1.059 & 1.063 \\
\hline & Men & 1.163 & 0.882 & 1.491 & 0.226 & 0.628 & 0.368 & 0.216 & 1.321 & 1.198 & 1.427 & 0.876 & 1.018 \\
\hline
\end{tabular}


Table 6.: Continued

\begin{tabular}{|c|c|c|c|c|c|c|c|c|c|c|c|c|c|}
\hline \multirow{3}{*}{ Role limitation due to emotional problems } & Overall & 0.740 & 0.526 & 0.832 & 0.398 & 0.593 & 0.605 & 0.597 & 1.498 & 1.363 & 1.380 & 1.342 & 1.562 \\
\hline & Women & 0.574 & 0.441 & 0.787 & 0.308 & 0.632 & 0.680 & 0.347 & 1.459 & 1.365 & 1.320 & 1.321 & 1.51 \\
\hline & Men & 0.946 & 0.600 & 0.820 & 0.513 & 0.482 & 0.471 & 0.988 & 1.364 & 1.162 & 1.307 & 1.191 & 1.429 \\
\hline \multirow{3}{*}{ Role limitation due to physical problems } & Overall & 0.913 & 0.669 & 1.128 & 0.398 & 0.273 & 0.428 & 0.248 & 1.532 & 1.525 & 1.478 & 1.176 & 1.31 \\
\hline & Women & 0.820 & 0.569 & 1.013 & 0.375 & 0.273 & 0.501 & 0.252 & 1.480 & 1.521 & 1.413 & 1.135 & 1.288 \\
\hline & Men & 1.043 & 0.841 & 1.305 & 0.401 & 0.246 & 0.230 & 0.203 & 1.410 & 1.287 & 1.409 & 1.082 & 1.178 \\
\hline \multirow{3}{*}{ Sexual function } & Overall & 1.183 & 0.638 & 0.757 & 0.183 & 0.307 & 0.381 & 0.309 & 1.514 & 1.367 & 1.546 & 1.161 & 1.495 \\
\hline & Women & 1.189 & 0.625 & 0.817 & 0.198 & 0.166 & 0.471 & 0.160 & 1.418 & 1.321 & 1.509 & 0.987 & 1.48 \\
\hline & Men & 0.894 & 0.598 & 0.648 & 0.077 & 0.889 & 0.434 & 0.724 & 1.472 & 1.204 & 1.311 & 1.460 & 1.20 \\
\hline \multirow{3}{*}{ Satisfaction with sexual function } & Overall & 1.217 & 0.683 & 0.850 & 0.258 & 0.292 & 0.365 & 0.422 & 1.491 & 1.258 & 1.527 & 1.210 & $1.50 c$ \\
\hline & Women & 1.213 & 0.579 & 0.775 & 0.322 & 0.038 & 0.288 & 0.165 & 1.451 & 1.307 & 1.531 & 1.064 & 1.44 \\
\hline & Men & 0.993 & 0.791 & 0.885 & 0.040 & 1.086 & 0.519 & 0.949 & 1.259 & 0.863 & 1.170 & 1.327 & 1.340 \\
\hline \multirow{3}{*}{ Social function } & Overall & 0.515 & 0.704 & 0.967 & 0.348 & 0.220 & 0.448 & 0.297 & 1.556 & 1.246 & 1.553 & 1.370 & 1.588 \\
\hline & Women & 0.345 & 0.470 & 0.765 & 0.190 & 0.106 & 0.177 & 0.159 & 1.540 & 1.335 & 1.620 & 1.176 & 1.67 \\
\hline & Men & 0.772 & 0.934 & 1.059 & $*$ & 0.642 & 0.685 & 0.439 & 1.372 & 0.932 & 1.223 & 1.522 & 1.217 \\
\hline
\end{tabular}

Note: All VIP scores that were shown to have a meaningful clinical influence are highlighted in red. All VIP scores from all the latent factors examined by the PLS analysis were in concordance with each other; therefore, all the values shown in the table are that of latent variable no. 1 (latent variables 2,3,4 and 5 are not shown). The sole exception is education's influence on men's social function, where latent factor no. 1 fell out of line from all other, concordant, and significant VIP scores for the rest of the latent factors.

${ }^{*}$ VIP scores for latent factors $1-5$ are as follows: $0.541,1.402,1.386,1.382,1.381$. 


\section{Acknowledgement}

I would like to thank to Professors László Vécsei and Péter Klivényi the former and present Heads of the Department of Neurology, University of Szeged for giving me the opportunity to work at the clinic and start my career as a neurologist. Furthermore I'd like to thank my supervisor, Associate Professor Krisztina Bencsik, MD, Ph.D., for letting me join the MS team in 2014 and for her years of supervision, perseverance, and continuous support of my work.

Additionally I wish to thank every co-author of the studies this work was based on, to every participant in the studies, and also to all my colleagues in the MS team.

Special thanks go to my friends and family, without whom this work would not have been born.

\section{References}

1. Trapp, B.D., et al., Axonal transection in the lesions of multiple sclerosis. N Engl J Med, 1998. 338(5): p. 278-85.

2. Lubetzki, C., et al., Remyelination in multiple sclerosis: from basic science to clinical translation. Lancet Neurol, 2020. 19(8): p. 678-688.

3. Bando, Y., Mechanism of demyelination and remyelination in multiple sclerosis. Clinical and Experimental Neuroimmunology, 2020. 11: p. 14-21.

4. Jean-Martin, C., Histologie de la sclérose en plaques. Extrait de la "Gazette des hôpitaux", 1868, p. 554 etc. Vol. 1 vol. (23 p.) ; In-8. 1868.

5. $\quad$ Compston, A., et al., McAlpine's Multiple Sclerosis. 4th ed. 2005.

6. $\quad$ Rudick, R.A., et al., Quality of life in multiple sclerosis. Comparison with inflammatory bowel disease and rheumatoid arthritis. Arch Neurol, 1992. 49(12): p. 1237-42.

7. $\quad$ Lublin, F.D., New multiple sclerosis phenotypic classification. Eur Neurol, 2014. 72 Suppl 1: p. 1-5.

8. Lublin, F.D. and S.C. Reingold, Defining the clinical course of multiple sclerosis: results of an international survey. National Multiple Sclerosis Society (USA) Advisory Committee on Clinical Trials of New Agents in Multiple Sclerosis. Neurology, 1996. 46(4): p. 907-11.

9. Lublin, F.D., et al., Defining the clinical course of multiple sclerosis: the 2013 revisions. Neurology, 2014. 83(3): p. 278-86.

10. Poser, C.M., et al., New diagnostic criteria for multiple sclerosis: guidelines for research protocols. Ann Neurol, 1983. 13(3): p. 227-31.

11. SCHUMACHER, G.A., et al., PROBLEMS OF EXPERIMENTAL TRIALS OF THERAPY IN MULTIPLE SCLEROSIS: REPORT BY THE PANEL ON THE EVALUATION OF EXPERIMENTAL TRIALS OF THERAPY IN MULTIPLE SCLEROSIS. Ann N Y Acad Sci, 1965. 122: p. 552-68.

12. McDonald, W.I., et al., Recommended diagnostic criteria for multiple sclerosis: guidelines from the International Panel on the diagnosis of multiple sclerosis. Ann Neurol, 2001. 50(1): p. 121-7.

13. Polman, C.H., et al., Diagnostic criteria for multiple sclerosis: 2005 revisions to the "McDonald Criteria". Ann Neurol, 2005. 58(6): p. 840-6. 
14. Polman, C.H., et al., Diagnostic criteria for multiple sclerosis: 2010 revisions to the McDonald criteria. Ann Neurol, 2011. 69(2): p. 292-302.

15. Thompson, A.J., et al., Diagnosis of multiple sclerosis: 2017 revisions of the McDonald criteria. Lancet Neurol, 2018. 17(2): p. 162-173.

16. Davda, N., E. Tallantyre, and N.P. Robertson, Early MRI predictors of prognosis in multiple sclerosis. J Neurol, 2019. 266(12): p. 3171-3173.

17. Kurtzke, J.F., Rating neurologic impairment in multiple sclerosis: an expanded disability status scale (EDSS). Neurology, 1983. 33(11): p. 1444-52.

18. Scalfari, A., et al., The natural history of multiple sclerosis: a geographically based study 10: relapses and long-term disability. Brain, 2010. 133(Pt 7): p. 1914-29.

19. Leray, E., et al., Evidence for a two-stage disability progression in multiple sclerosis. Brain, 2010. 133(Pt 7): p. 1900-13.

20. Amato, M.P., et al., Benign multiple sclerosis: cognitive, psychological and social aspects in a clinical cohort. J Neurol, 2006. 253(8): p. 1054-9.

21. Correale, J., I. Peirano, and L. Romano, Benign multiple sclerosis: a new definition of this entity is needed. Mult Scler, 2012. 18(2): p. 210-8.

22. Sayao, A.L., V. Devonshire, and H. Tremlett, Longitudinal follow-up of "benign" multiple sclerosis at 20 years. Neurology, 2007. 68(7): p. 496-500.

23. Ton, A.M.M., C.C.F. Vasconcelos, and R.M.P. Alvarenga, Benign multiple sclerosis: aspects of cognition and neuroimaging. Arq Neuropsiquiatr, 2017. 75(6): p. 394-401.

24. Lorscheider, J., et al., Defining secondary progressive multiple sclerosis. Brain, 2016. 139(Pt 9): p. 2395-405.

25. Correale, J., et al., Progressive multiple sclerosis: from pathogenic mechanisms to treatment. Brain, 2017. 140(3): p. 527-546.

26. Purmonen, T., et al., Impact of multiple sclerosis phenotypes on burden of disease in Finland. J Med Econ, 2020. 23(2): p. 156-165.

27. Montalban, X., et al., ECTRIMS/EAN Guideline on the pharmacological treatment of people with multiple sclerosis. Mult Scler, 2018. 24(2): p. 96-120.

28. Kingwell, E., et al., Incidence and prevalence of multiple sclerosis in Europe: a systematic review. BMC Neurol, 2013. 13: p. 128.

29. Phadke, J.G., Survival pattern and cause of death in patients with multiple sclerosis: results from an epidemiological survey in north east Scotland. J Neurol Neurosurg Psychiatry, 1987. 50(5): p. 523-31.

30. Sandi, D., et al., Mortality in Hungarian patients with multiple sclerosis between 1993 and 2013. J Neurol Sci, 2016. 367: p. 329-32.

31. Brønnum-Hansen, H., N. Koch-Henriksen, and E. Stenager, Trends in survival and cause of death in Danish patients with multiple sclerosis. Brain, 2004. 127(Pt 4): p. 84450.

32. Leray, E., et al., Long-term survival of patients with multiple sclerosis in West France. Mult Scler, 2007. 13(7): p. 865-74.

33. Kingwell, E., et al., Relative mortality and survival in multiple sclerosis: findings from British Columbia, Canada. J Neurol Neurosurg Psychiatry, 2012. 83(1): p. 61-6.

34. Lunde, H.M.B., et al., Survival and cause of death in multiple sclerosis: a 60-year longitudinal population study. J Neurol Neurosurg Psychiatry, 2017. 88(8): p. 621-625.

35. Grytten Torkildsen, N., et al., Survival and cause of death in multiple sclerosis: results from a 50-year follow-up in Western Norway. Mult Scler, 2008. 14(9): p. 1191-8.

36. Feinstein, A. and B. Pavisian, Multiple sclerosis and suicide. Mult Scler, 2017. 23(7): p. 923-927.

37. Brønnum-Hansen, H., et al., Suicide among Danes with multiple sclerosis. J Neurol Neurosurg Psychiatry, 2005. 76(10): p. 1457-9. 
38. Shen, Q., et al., Association between suicide and multiple sclerosis: An updated metaanalysis. Mult Scler Relat Disord, 2019. 34: p. 83-90.

39. Ruet, A., et al., Cognitive impairment, health-related quality of life and vocational status at early stages of multiple sclerosis: a 7-year longitudinal study. J Neurol, 2013. 260(3): p. 776-84.

40. Lanzillo, R., et al., Quality of life and cognitive functions in early onset multiple sclerosis. Eur J Paediatr Neurol, 2016. 20(1): p. 158-63.

41. Solaro, C., G. Gamberini, and F.G. Masuccio, Depression in Multiple Sclerosis: Epidemiology, Aetiology, Diagnosis and Treatment. CNS Drugs, 2018. 32(2): p. 117133.

42. Amato, M.P., et al., Quality of life in multiple sclerosis: the impact of depression, fatigue and disability. Mult Scler, 2001. 7(5): p. 340-4.

43. Amato, M.P., et al., Association of MRI metrics and cognitive impairment in radiologically isolated syndromes. Neurology, 2012. 78(5): p. 309-14.

44. Glanz, B.I., et al., Cognitive dysfunction in patients with clinically isolated syndromes or newly diagnosed multiple sclerosis. Mult Scler, 2007. 13(8): p. 1004-10.

45. Ben Ari Shevil, E., et al., How are cognitive impairment, fatigue and signs of depression related to participation in daily life among persons with multiple sclerosis? Disabil Rehabil, 2014. 36(23): p. 2012-8.

46. Chiaravalloti, N.D. and J. DeLuca, Cognitive impairment in multiple sclerosis. Lancet Neurol, 2008. 7(12): p. 1139-51.

47. Sandi, D., et al., Prevalence of cognitive impairment among Hungarian patients with relapsing-remitting multiple sclerosis and clinically isolated syndrome. Mult Scler Relat Disord, 2017. 17: p. 57-62.

48. $\quad$ Langdon, D.W., Cognition in multiple sclerosis. Curr Opin Neurol, 2011. 24(3): p. 2449.

49. Benedict, R.H., et al., Predicting quality of life in multiple sclerosis: accounting for physical disability, fatigue, cognition, mood disorder, personality, and behavior change. J Neurol Sci, 2005. 231(1-2): p. 29-34.

50. Lobentanz, I.S., et al., Factors influencing quality of life in multiple sclerosis patients: disability, depressive mood, fatigue and sleep quality. Acta Neurol Scand, 2004. 110(1): p. 6-13.

51. Janardhan, V. and R. Bakshi, Quality of life in patients with multiple sclerosis: the impact of fatigue and depression. J Neurol Sci, 2002. 205(1): p. 51-8.

52. Hungarian Central Statistical Office.

53. www.ksh.hu. https://www.ksh.hu/docs/hun/xstadat/xstadat_eves/i_wdsd004c.html, Last accessed: 05, December, 2019

54. Bencsik, K., et al., [The Multiple Sclerosis Registry of Szeged]. Ideggyogy Sz, 2017. 70(9-10): p. 301-306.

55. Filippi, M., et al., MRI criteria for the diagnosis of multiple sclerosis: MAGNIMS consensus guidelines. Lancet Neurol, 2016. 15(3): p. 292-303.

56. Langdon, D.W., et al., Recommendations for a Brief International Cognitive Assessment for Multiple Sclerosis (BICAMS). Mult Scler, 2012. 18(6): p. 891-8.

57. Dusankova, J.B., et al., Cross cultural validation of the Minimal Assessment of Cognitive Function in Multiple Sclerosis (MACFIMS) and the Brief International Cognitive Assessment for Multiple Sclerosis (BICAMS). Clin Neuropsychol, 2012. 26(7): p. 1186-200.

58. Beck, A.T.S., R.A. Brown, G.K., Manual for the Beck Depression Inventory-II. . 1996: San Antonio. 
59. Losonczi, E., et al., Validation of the Fatigue Impact Scale in Hungarian patients with multiple sclerosis. Qual Life Res, 2011. 20(2): p. 301-6.

60. Füvesi, J., et al., Cross-cultural adaptation and validation of the 'Multiple Sclerosis Quality of Life Instrument' in Hungarian. Mult Scler, 2008. 14(3): p. 391-8.

61. Ware, J.E., SF-36 health survey update. Spine (Phila Pa 1976), 2000. 25(24): p. 31309.

62. Perenboom, R.J. and A.M. Chorus, Measuring participation according to the International Classification of Functioning, Disability and Health (ICF). Disabil Rehabil, 2003. 25(11-12): p. 577-87.

63. Brown, C.A., et al., SF-36 includes less Parkinson Disease (PD)-targeted content but is more responsive to change than two PD-targeted health-related quality of life measures. Qual Life Res, 2009. 18(9): p. 1219-37.

64. Guilfoyle, M.R., H. Seeley, and R.J. Laing, The Short Form 36 health survey in spine disease--validation against condition-specific measures. Br J Neurosurg, 2009. 23(4): p. 401-5.

65. Wang, R., et al., Health related quality of life measured by SF-36: a population-based study in Shanghai, China. BMC Public Health, 2008. 8: p. 292.

66. Lim, L.L., S.A. Seubsman, and A. Sleigh, Thai SF-36 health survey: tests of data quality, scaling assumptions, reliability and validity in healthy men and women. Health Qual Life Outcomes, 2008. 6: p. 52.

67. Tóth, E., et al., Gray Matter Atrophy Is Primarily Related to Demyelination of Lesions in Multiple Sclerosis: A Diffusion Tensor Imaging MRI Study. Front Neuroanat, 2017. 11: p. 23.

68. Wold, S., E. Johansson, and M. Cocchi, PLS - Partial least squares projections to latent structures., in 3D QSAR in drug design K. H., Editor. 1994, ESCOM Science Publishers: Leiden. p. 523-550.

69. Benjak, T., et al., Prevalence of multiple sclerosis in Croatia: data from national and non-governmental organization registries. Croat Med J, 2018. 59(2): p. 65-70.

70. Kapica-Topczewska, K., et al., Prevalence of multiple sclerosis in Poland. Mult Scler Relat Disord, 2018. 21: p. 51-55.

71. Salhofer-Polanyi, S., et al., Epidemiology of Multiple Sclerosis in Austria. Neuroepidemiology, 2017. 49(1-2): p. 40-44.

72. Zsiros, V., et al., Prevalence of multiple sclerosis in Csongrád County, Hungary. Acta Neurol Scand, 2014. 130(5): p. 277-82.

73. Flemmen, H., et al., Prevalence of multiple sclerosis in rural and urban districts in Telemark county, Norway. Mult Scler Relat Disord, 2020. 45: p. 102352.

74. Brola, W., et al., Prevalence and incidence of multiple sclerosis in central Poland, 20102014. BMC Neurol, 2016. 16(1): p. 134.

75. Iljicsov, A., et al., Incidence and prevalence of multiple sclerosis in Hungary based on record linkage of nationwide multiple healthcare administrative data. PLoS One, 2020. 15(7): p. e0236432.

76. Bencsik, K., et al., The prevalence of multiple sclerosis, distribution of clinical forms of the disease and functional status of patients in Csongrád County, Hungary. Eur Neurol, 2001. 46(4): p. 206-9.

77. Benito-León, J., Multiple sclerosis: is prevalence rising and if so why? Neuroepidemiology, 2011. 37(3-4): p. 236-7.

78. Koch-Henriksen, N., et al., Incidence of MS has increased markedly over six decades in Denmark particularly with late onset and in women. Neurology, 2018. 90(22): p. e1954-e1963. 
79. Grytten, N., Ø. Torkildsen, and K.M. Myhr, Time trends in the incidence and prevalence of multiple sclerosis in Norway during eight decades. Acta Neurol Scand, 2015. 132(199): p. 29-36.

80. Hungarian Central Statistical Office. 2019: Hungary.

81. Alpar, A. 2016. p. https://www.fokuszpont.dohanyzasvissza szoritasa.hu/sites/default/files/dohanyzas_visszaszoritasa_2016_ dohanyzas_fokuszpont.pdf.

82. Hillert, J. and L. Stawiarz, The Swedish MS registry-clinical support tool and scientific resource. Acta Neurol Scand, 2015. 132(199): p. 11-9.

83. Laakso, S.M., et al., Multiple sclerosis in Finland 2018-Data from the national register. Acta Neurol Scand, 2019. 140(5): p. 303-311.

84. Mellinger, S., et al., Multiple sclerosis prevalence in Salta City, Argentina. Mult Scler Relat Disord, 2018. 25: p. 212-215.

85. Pirttisalo, A.L., M. Soilu-Hänninen, and J.O.T. Sipilä, Multiple sclerosis epidemiology in Finland: Regional differences and high incidence. Acta Neurol Scand, 2019. 139(4): p. 353-359.

86. Trojano, M., et al., The Italian multiple sclerosis register. Neurol Sci, 2019. 40(1): p. 155-165.

87. Urru, S.A., et al., Prevalence of multiple sclerosis in Sardinia: A systematic crosssectional multi-source survey. Mult Scler, 2020. 26(3): p. 372-380.

88. Ricardo, A., et al., Clinical and demographic characteristics of primary progressive multiple sclerosis in Argentina: Argentinean registry cohort study (RelevarEM). Neurol Sci, 2020.

89. Ricardo, A., et al., Correction to: Clinical and demographic characteristics of primary progressive multiple sclerosis in Argentina: Argentinean registry cohort study (RelevarEM). Neurol Sci, 2020.

90. Rojas, J.I., et al., Multiple sclerosis and neuromyelitis optica spectrum disorders in Argentina: comparing baseline data from the Argentinean MS Registry (RelevarEM). Neurol Sci, 2020. 41(6): p. 1513-1519.

91. Kalincik, T., et al., Towards personalized therapy for multiple sclerosis: prediction of individual treatment response. Brain, 2017. 140(9): p. 2426-2443.

92. Lankhorst, G.J., et al., Quality of life in multiple sclerosis: the disability and impact profile (DIP). J Neurol, 1996. 243(6): p. 469-74.

93. Ford, H.L., et al., Health status and quality of life of people with multiple sclerosis. Disabil Rehabil, 2001. 23(12): p. 516-21.

94. Khan, F., et al., Multiple sclerosis: disability profile and quality of life in an Australian community cohort. Int J Rehabil Res, 2006. 29(2): p. 87-96.

95. Ziemssen, T., Multiple sclerosis beyond EDSS: depression and fatigue. J Neurol Sci, 2009. 277 Suppl 1: p. S37-41.

96. Pittion-Vouyovitch, S., et al., Fatigue in multiple sclerosis is related to disability, depression and quality of life. J Neurol Sci, 2006. 243(1-2): p. 39-45.

97. Motl, R.W., et al., Physical activity and quality of life in multiple sclerosis: intermediary roles of disability, fatigue, mood, pain, self-efficacy and social support. Psychol Health Med, 2009. 14(1): p. 111-24.

98. Hoogs, M., et al., Cognition and physical disability in predicting health-related quality of life in multiple sclerosis. Int J MS Care, 2011. 13(2): p. 57-63.

99. Campbell, J., et al., Cognitive impairment among patients with multiple sclerosis: associations with employment and quality of life. Postgrad Med J, 2017. 93(1097): p. 143-147. 
100. Merkelbach, S., H. Sittinger, and J. Koenig, Is there a differential impact of fatigue and physical disability on quality of life in multiple sclerosis? J Nerv Ment Dis, 2002. 190(6): p. 388-93.

101. Twork, S., et al., Disability status and quality of life in multiple sclerosis: non-linearity of the Expanded Disability Status Scale (EDSS). Health Qual Life Outcomes, 2010. 8: p. 55.

102. Corfield, F. and D. Langdon, A Systematic Review and Meta-Analysis of the Brief Cognitive Assessment for Multiple Sclerosis (BICAMS). Neurol Ther, 2018. 7(2): p. 287-306.

103. Benedict, R.H.B., et al., Cognitive impairment in multiple sclerosis: clinical management, MRI, and therapeutic avenues. Lancet Neurol, 2020. 19(10): p. 860-871.

104. Patti, F., et al., Prevalence and incidence of cognitive impairment in multiple sclerosis: a population-based survey in Catania, Sicily. J Neurol, 2015. 262(4): p. 923-30.

105. Füvesi, J., et al., Factors influencing the health-related quality of life in Hungarian multiple sclerosis patients. J Neurol Sci, 2010. 293(1-2): p. 59-64.

106. Sander, L., J. Kugler, and B. Elsner, [The influence of multiple sclerosis-related symptoms on health-related quality of life]. Fortschr Neurol Psychiatr, 2020.

107. Shohani, M., et al., The effect of yoga on the quality of life and fatigue in patients with multiple sclerosis: A systematic review and meta-analysis of randomized clinical trials. Complement Ther Clin Pract, 2020. 39: p. 101087.

108. Miller, D.M. and R. Allen, Quality of life in multiple sclerosis: determinants, measurement, and use in clinical practice. Curr Neurol Neurosci Rep, 2010. 10(5): p. 397-406.

109. Mitchell, A.J., et al., Quality of life and its assessment in multiple sclerosis: integrating physical and psychological components of wellbeing. Lancet Neurol, 2005. 4(9): p. 55666.

110. Glanz, B.I., et al., The association between cognitive impairment and quality of life in patients with early multiple sclerosis. J Neurol Sci, 2010. 290(1-2): p. 75-9.

111. Benedict, R.H., et al., Personality disorder in multiple sclerosis correlates with cognitive impairment. J Neuropsychiatry Clin Neurosci, 2001. 13(1): p. 70-6.

112. Benedict, R.H., et al., Prediction of neuropsychological impairment in multiple sclerosis: comparison of conventional magnetic resonance imaging measures of atrophy and lesion burden. Arch Neurol, 2004. 61(2): p. 226-30.

113. Schwartz, C.E., et al., Cognitive reserve and patient-reported outcomes in multiple sclerosis. Mult Scler, 2013. 19(1): p. 87-105.

114. Greer, J.M. and P.A. McCombe, Role of gender in multiple sclerosis: clinical effects and potential molecular mechanisms. J Neuroimmunol, 2011. 234(1-2): p. 7-18.

115. Zaffaroni, M. and A. Ghezzi, The prognostic value of age, gender, pregnancy and endocrine factors in multiple sclerosis. Neurol Sci, 2000. 21(4 Suppl 2): p. S857-60.

116. Miller, A. and S. Dishon, Health-related quality of life in multiple sclerosis: The impact of disability, gender and employment status. Qual Life Res, 2006. 15(2): p. 259-71.

117. Amato, M.P., et al., Disease-modifying drugs can reduce disability progression in relapsing multiple sclerosis. Brain, 2020.

118. Casetta, I., et al., Gender differences in health-related quality of life in multiple sclerosis. Mult Scler, 2009. 15(11): p. 1339-46.

119. A.W, B., et al. What Jobs are Being Done at Home During the COVID-19 Crisis? Evidence from Firm-Level Surveys. 2020.

120. Zumbrun, J. Is the Gender Pay Gap Closing or Has Progress Stalled? 2014.

121. Barnwell, A.M. and D.J. Kavanagh, Prediction of psychological adjustment to multiple sclerosis. Soc Sci Med, 1997. 45(3): p. 411-8. 
122. Duval, M.L., Psychosocial metaphors of physical distress among MS patients. Soc Sci Med, 1984. 19(6): p. 635-8.

123. Király, A., et al., Male brain ages faster: the age and gender dependence of subcortical volumes. Brain Imaging Behav, 2016. 10(3): p. 901-10.

124. Eshaghi, A., et al., Progression of regional grey matter atrophy in multiple sclerosis. Brain, 2018. 141(6): p. 1665-1677.

125. Genovese, A.V., et al., Atrophied Brain T2 Lesion Volume at MRI Is Associated with Disability Progression and Conversion to Secondary Progressive Multiple Sclerosis. Radiology, 2019. 293(2): p. 424-433.

126. Hakim, E.A., et al., The social impact of multiple sclerosis--a study of 305 patients and their relatives. Disabil Rehabil, 2000. 22(6): p. 288-93.

127. Nortvedt, M.W., et al., Reduced quality of life among multiple sclerosis patients with sexual disturbance and bladder dysfunction. Mult Scler, 2001. 7(4): p. 231-5.

128. Quality of life indicators - education. 2019.

129. Yalachkov, Y., et al., Determinants of quality of life in relapsing-remitting and progressive multiple sclerosis. Mult Scler Relat Disord, 2019. 30: p. 33-37.

130. Nourbakhsh, B., L. Julian, and E. Waubant, Fatigue and depression predict quality of life in patients with early multiple sclerosis: a longitudinal study. Eur J Neurol, 2016. 23(9): p. 1482-6.

131. Brissart, H., et al., Memory improvement in multiple sclerosis after an extensive cognitive rehabilitation program in groups with a multicenter double-blind randomized trial. Clin Rehabil, 2020. 34(6): p. 754-763. 